\title{
Measuring effective electroweak couplings in single top quark production at the CERN LHC
}

\author{
D. Espriu* and J. Manzano \\ Departament d'Estructura i Constituents, de la Matèria and IFAE, Universitat de Barcelona, Diagonal, 647, E-08028 Barcelona, Spain
}

(Received 11 July 2001; published 8 March 2002)

\begin{abstract}
We study the mechanism of single top quark production at the CERN LHC in the framework of an effective electroweak Lagrangian, analyzing the sensitivity of different observables to the magnitude of the effective couplings that parametrize new physics beyond the standard model. The observables relevant to the distinction between left and right effective couplings involve in practice the measurement of the spin of the top quark and this can be achieved only indirectly by measuring the angular distribution of its decay products. We show that the presence of effective right-handed couplings implies that the top quark is not in a pure spin state. A unique spin basis is singled out which allows one to connect the top decay products angular distribution with the polarized top differential cross section. We present a complete analytical expression of the differential polarized cross section of the relevant perturbative subprocess including general effective couplings. The mass of the bottom quark, which actually turns out to be more relevant than naively expected, is retained. Finally we analyze different aspects of the total cross section relevant to the measurement of new physics through the effective couplings. The above analysis also applies to top antiquark production in a straightforward way.
\end{abstract}

DOI: 10.1103/PhysRevD.65.073005

PACS number(s): 12.15.Ff, 14.65.Ha

\section{INTRODUCTION}

The standard model of electroweak and strong interactions has been, to this day, tested to a remarkable degree of accuracy, particularly in what concerns the neutral current sector. However it is clear that it suffers from several theoretical drawbacks (naturalness, triviality, ... ) making it conceivable that it should be considered as an effective theory valid only at low energies ( $\lesssim 1 \mathrm{TeV})$. With the current limit on the Higgs boson mass already placed at $113.5 \mathrm{GeV}$ [1] and no clear evidence for the existence of an elementary scalar (despite much controversy regarding the results of the last days of the CERN $e^{+} e^{-}$collider LEP) it makes sense to envisage an alternative to the minimal standard model described by an effective theory without any physical light scalar fields. This is in spite of the seemingly good agreement between experiment and radiative corrections computed in the framework of the minimal standard model (see [2] however). This effective theory should contain an infinite set of effective operators, of increasing dimensionality, compatible with the electroweak and strong symmetries $S U(3)_{c}$ $\times S U(2)_{L} \times U(1)_{Y}$ and their coefficients would parametrize physics beyond the standard model. In this framework [3] one can describe the low energy physics of theories exhibiting the pattern of symmetry breaking $S U(2)_{L} \times U(1)_{Y}$ $\rightarrow U(1)_{e m}$ with full generality, in the understanding that this approach is useful as long as those particles not explicitly included in the effective Lagrangian are much heavier than the scale of energies at which the effective Lagrangian is to be used.

In this work we plan to investigate the new features that physics beyond the standard model may introduce in the production of the top quarks (or top antiquarks) through $W$-gluon fusion at the CERN Large Hadron Collider (LHC).

\footnotetext{
*Electronic address: espriu@ecm.ub.es

†Electronic address: manzano@ecm.ub.es
}

When describing the appropriate effective vertex in the effective Lagrangian language, we will keep only the leading nonuniversal (i.e., not appearing in the standard model) effective operators in the low energy expansion. They correspond to the operators of dimension four, which were first classified by Appelquist $e t$ al. [4]. These operators realize the $S U(2)_{L} \times U(1)_{Y}$ symmetry non-linearly and are thus characteristic of strongly coupled theories and, strictly speaking, are absent in the minimal standard model and modifications thereof containing only light fields, such as supersymmetric extensions, where all degrees of freedom are weakly interacting and need to be included explicitly. When particularizing to interactions involving the $W, Z$ bosons, the operators present in the effective electroweak Lagrangian induce effective vertices coupling the gauge boson to the matter fields (see e.g. [5])

$$
\begin{aligned}
& -\frac{e}{4 c_{W^{S} S_{W}}} \bar{f} \gamma^{\mu}\left(\kappa_{L}^{N C} L+\kappa_{R}^{N C} R\right) Z_{\mu} f-\frac{e}{s_{W}} \bar{f} \gamma^{\mu} \\
& \times\left(\kappa_{L}^{C C} L+\kappa_{R}^{C C} R\right) \frac{\tau^{-}}{2} W_{\mu}^{+} f+\text { H.c. }
\end{aligned}
$$

Other possible effects are not physically observable, as we shall see in a moment. In practical terms, LHC will set bounds on these effective $W$ vertices, and therefore our results are also relevant in a broader phenomenological context as a way to bound $\kappa_{L}$ and $\kappa_{R}$, without any need to appeal to an underlying effective Lagrangian describing a specific model of symmetry breaking. Of course one then looses the power of an effective Lagrangian, namely a well defined set of counting rules and the ability to relate different processes.

Another point to note is that, even in the minimal standard model, radiative corrections induce modifications in the vertices. Assuming a smooth dependence in the external momenta these form factors can be expanded in powers of momenta. At the lowest order in the derivative expansion, the effect of radiative corrections can be encoded in the effective 


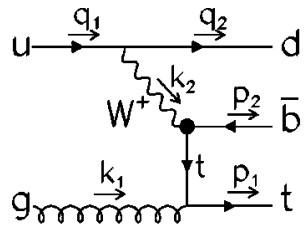

(a)

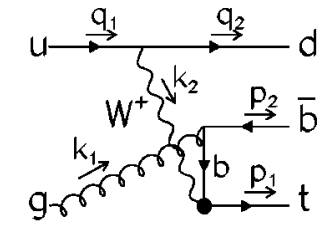

(b)
FIG. 1. Feynman diagrams contributing to single top production subprocess. In this case we have a $d$ as the spectator quark.

vertices $\kappa_{L}$ and $\kappa_{R}$. Thus these effective vertices take well defined, calculable values in the minimal standard model, and any deviation from these values (which, incidentally, have not been fully determined in the standard model yet) would indicate the presence of new physics in the matter sector. The extent to what LHC can set direct bounds on the effective vertices, in particular on those involving the third generation, is highly relevant to constraint physics beyond the standard model in a direct way. This paper is devoted to such an analysis in charged processes involving a top quark at the LHC.

At the LHC energy $(14 \mathrm{TeV})$ the dominant mechanism of top production, with a cross section of $800 \mathrm{pb}$ [6], is gluongluon fusion. This mechanism has nothing to do with the electroweak sector and thus is not the most adequate for our purposes (although is the one producing most of the tops quarks and thus its consideration becomes necessary in order to study the top couplings through their decay, which will not be our main interest here, and also as a background to the process we shall be interested in).

Electroweak physics enters the game in single top production. (For a recent review see e.g. [7].) At LHC energies the (by far) dominant electroweak subprocess contributing to single top production is given by a gluon $(g)$ coming from one proton and a light quark or anti-quark coming from the other (this process is also called $t$-channel production $[8,9]$ ). This process is depicted in Figs. 1 and 2, where light $u$-type quarks or $\bar{d}$-type antiquarks are extracted from the proton, respectively. These quarks then radiate a $W$ whose effective couplings are the object of our interest. The total cross section for this process at the LHC is estimated to be $250 \mathrm{pb}$ [9], to be compared to $50 \mathrm{pb}$ for the associated production with a $W^{+}$boson and a $b$-quark extracted from the sea of the proton, and $10 \mathrm{pb}$ corresponding to quark-quark fusion ( $s$-channel production). For comparison, at the Tevatron (2 $\mathrm{GeV}$ ) the cross section for $W$-gluon fusion is $2.5 \mathrm{pb}$, so the production of top quarks through this particular subprocess is

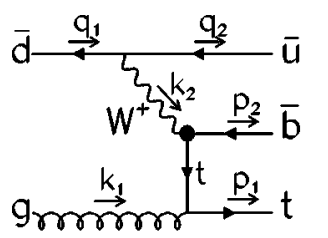

(a)

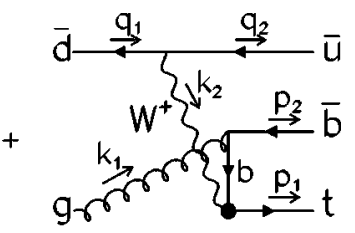

(b)
FIG. 2. Feynman diagrams contributing single top production subprocess. In this case we have a $\bar{u}$ as the spectator quark. copious at the LHC. Monte Carlo simulations including the analysis of the top decay products indicate that this process can be analyzed in detail at the LHC and traditionally has been regarded as the most important one for our purposes.

In a proton-proton collision a bottom-quark-topantiquark pair is also produced, through analogous subprocesses. At any rate qualitative results are very similar to those corresponding to top production, from where the cross sections can be easily derived doing the appropriate changes.

In the context of effective theories, the contribution from operators of dimension five to top production via longitudinal vector boson fusion was estimated some time ago in [10], although the study was by no means complete. It should be mentioned that $t, \bar{t}$ pair production through this mechanism is very much masked by the dominant mechanism of gluongluon fusion, while single top production, through $W Z$ fusion, is expected to be much suppressed compared to the mechanism presented in this paper, the reason being that both vertices are electroweak in the process discussed in [10], and that operators of dimension five are expected to be suppressed, at least at moderate energies, by some large mass scale. The contribution from dimension four operators as such has not, to our knowledge, been considered before, although the potential for single top production for measuring the Cabibbo-Kobayashi-Maskawa (CKM) matrix element $K_{t b}$ has to some extent been analyzed in the past (see e.g. $[9,11])$.

\section{EFFECTIVE COUPLINGS AND OBSERVABLES}

Including family mixing and, possibly, $C P$ violation, the complete set of dimension four effective operators which may contribute to the top effective couplings and are relevant for the present discussion is $[4,12,13]$

$$
\begin{aligned}
& \mathcal{L}_{L}^{1}=i \overline{\mathrm{f}} M_{L}^{1} \gamma^{\mu} U\left(D_{\mu} U\right)^{\dagger} L \mathrm{f}+\text { H.c., } \\
& \mathcal{L}_{L}^{2}=i \overline{\mathrm{f}} M_{L}^{2} \gamma^{\mu}\left(D_{\mu} U\right) \tau^{3} U^{\dagger} L \mathrm{f}+\text { H.c., } \\
& \mathcal{L}_{L}^{3}=i \overline{\mathrm{f}} M_{L}^{3} \gamma^{\mu} U \tau^{3} U^{\dagger}\left(D_{\mu} U\right) \tau^{3} U^{\dagger} L \mathrm{f}+\text { H.c. } \\
& \mathcal{L}_{L}^{4}=i \overline{\mathrm{f}} M_{L}^{4} \gamma^{\mu} U \tau^{3} U^{\dagger} D_{\mu}^{L} L \mathrm{f}+\text { H.c. }
\end{aligned}
$$

and

$$
\begin{aligned}
& \mathcal{L}_{R}^{1}=i \overline{\mathrm{f}} M_{R}^{1} \gamma^{\mu} U^{\dagger}\left(D_{\mu} U\right) R \mathrm{f}+\text { H.c., } \\
& \mathcal{L}_{R}^{2}=i \overline{\mathrm{f}} M_{R}^{2} \gamma^{\mu} \tau^{3} U^{\dagger}\left(D_{\mu} U\right) R \mathrm{f}+\text { H.c., } \\
& \mathcal{L}_{R}^{3}=i \overline{\mathrm{f}} M_{R}^{3} \gamma^{\mu} \tau^{3} U^{\dagger}\left(D_{\mu} U\right) \tau^{3} R \mathrm{f}+\text { H.c. },
\end{aligned}
$$

where $L=\left(1-\gamma^{5}\right) / 2, R=\left(1+\gamma^{5}\right) / 2$ are the left and right projectors, the matrices $M$ have family indices only, and the above set is written the nonphysical "weak" basis (that is, with matter fields transforming as irreducible representations of the gauge group) [13]. The unitary matrix $U$ contains the 
three Goldstone bosons associated to the breakdown of the global symmetry $S U(2)_{L} \times S U(2)_{R}$ down to $S U(2)_{V}$. The derivatives appearing in the effective operators are given by

$$
\begin{aligned}
& D_{\mu} U=\partial_{\mu} U+i g \frac{\tau}{2} \cdot W_{\mu} U-i g^{\prime} U \frac{\tau^{3}}{2} B_{\mu} \\
& D_{\mu}^{L} \mathrm{f}=\left(\partial_{\mu}+i g \frac{\tau}{2} \cdot W_{\mu}+i g^{\prime}\left(Q-\frac{\tau^{3}}{2}\right) B_{\mu}+i g_{s} \frac{\lambda}{2} \cdot G_{\mu}\right) \mathrm{f} \\
& D_{\mu}^{R} \mathrm{f}=\left(\partial_{\mu}+i g^{\prime} Q B_{\mu}+i g_{s} \frac{\lambda}{2} \cdot G_{\mu}\right) \mathrm{f}
\end{aligned}
$$

where $f$ is a weak doublet of matter fields with family indices also. In addition, one has the following "universal" terms

$$
\begin{aligned}
\mathcal{L}_{S M}= & i \overline{\mathrm{f}} \gamma^{\mu}\left[M^{L} D_{\mu}^{L} L+\left(\tau^{u} M_{u}^{R}+\tau^{d} M_{d}^{R}\right) D_{\mu}^{R} R\right] \mathrm{f} \\
& -\overline{\mathrm{f}}\left(U\left(\tau^{u} \tilde{y}_{u}^{f}+\tau^{d} \tilde{y}_{d}^{f}\right) R+\left(\tau^{u} \tilde{y}_{u}^{f \dagger}+\tau^{d} \tilde{y}_{d}^{f \dagger}\right) U^{\dagger} L\right) \mathrm{f},
\end{aligned}
$$

which are present in the standard model. In Eq. (5) we allow for general couplings $M_{L}, M_{R}^{(u, d)}$; in the standard model these couplings can be renormalized away via a change of basis, but in more general theories they leave traces in other operators not present in the standard model [13].

In [13] it was also shown that when we diagonalize the mass matrix present in Eq. (5) via a redefinition of the matter fields $(\mathrm{f} \rightarrow f$ ) we change also the structure of operators (2), (3). Taking that into account, the contribution to the different gauge boson-fermion-fermion vertices is as follows:

$$
\begin{aligned}
\mathcal{L}_{b f f}= & -g_{s} \bar{f} \gamma^{\mu}\left(a_{L} L+a_{R} R\right) \frac{\lambda}{2} \cdot \mathbf{G}_{\mu} f, \\
& -e \bar{f} \gamma^{\mu}\left(b_{L} L+b_{R} R\right) A_{\mu} f, \\
& -\frac{e}{2 c_{W} s_{W}} \bar{f} \gamma^{\mu}\left[\left(c_{L}^{u} \tau^{u}+c_{L}^{d} \tau^{d}\right) L+\left(c_{R}^{u} \tau^{u}+c_{R}^{d} \tau^{d}\right) R\right] Z_{\mu} f \\
& -\frac{e}{s_{W}} \bar{f} \gamma^{\mu}\left[\left(d_{L} L+d_{R} R\right) \frac{\tau^{-}}{2} W_{\mu}^{+}\right. \\
& \left.+\left(d_{L}^{\dagger} L+d_{R}^{\dagger} R\right) \frac{\tau^{+}}{2} W_{\mu}^{-}\right] f,
\end{aligned}
$$

where $\tau^{u}$ and $\tau^{d}$ are the up and down projectors and $f$ represents the matter fields in the physical, diagonal basis. It can be shown that once the all the renormalization (vertex, CKM elements, wave function) countertems are taken into account [13] we obtain $a_{L, R}=1, b_{L, R}=Q$; i.e., we have no contribution from the effective operators to the vertices of the gluon and photon. For the $Z$ couplings we get instead

$$
\begin{aligned}
c_{L}^{u}= & 1-2 Q s_{W}^{2}-N^{1}-N^{1 \dagger}+N^{2 \dagger}+N^{2}+N^{3}+N^{3 \dagger}, \\
c_{L}^{d}= & -1-2 Q s_{W}^{2}+K^{\dagger}\left(N^{1}+N^{1 \dagger}+N^{2 \dagger}+N^{2}-N^{3}\right. \\
& \left.-N^{3 \dagger}\right) K,
\end{aligned}
$$

$$
\begin{aligned}
& c_{R}^{u}=-2 s_{W}^{2} Q+\tilde{M}^{1}+\tilde{M}^{1 \dagger}+\tilde{M}^{2}+\tilde{M}^{2 \dagger}+\tilde{M}^{3}+\tilde{M}^{3 \dagger}, \\
& c_{R}^{d}=-2 s_{W}^{2} Q+\tilde{M}^{1}+\tilde{M}^{1 \dagger}-\tilde{M}^{2}-\tilde{M}^{2 \dagger}-\tilde{M}^{3}-\tilde{M}^{3 \dagger},
\end{aligned}
$$

where $K$ is the CKM matrix, and the matrices $N$ 's and $\widetilde{M}$ 's are redefined matrices according to the results of [13] [the exact relation of these matrices to the $M$ 's of Eqs. (2), (3) has no relevance for the present discussion]. Finally for the charged couplings we have

$$
\begin{aligned}
& d_{L}=K+\left(-N^{1}-N^{1 \dagger}+N^{2}-N^{2 \dagger}-N^{3}-N^{3 \dagger}+N^{4}-N^{4 \dagger}\right) K, \\
& d_{R}=\widetilde{M}^{1}+\widetilde{M}^{1 \dagger}+\widetilde{M}^{2}-\widetilde{M}^{2 \dagger}-\widetilde{M}^{3}-\widetilde{M}^{3 \dagger} .
\end{aligned}
$$

Since the set of operators (2), (3) is the most general one allowed by general requirements of gauge invariance, locality and hermiticity; it is clear that radiative corrections, when expanded in powers of $p^{2}$, can be incorporated into them. In fact, such an approach has proven to be very fruitful in the past. Once everything is included we are allowed to identify the couplings $d_{L, R}$ with $\kappa_{L R}^{C C}$. In this paper we shall be concerned with the bounds that the LHC experiments will be able to set on the couplings $\kappa_{L R}^{C C}$, more specifically on the entries $t j$ of these matrices (those involving the top). In the rest of the article we do not consider mixing and we consider nontree-level and new physics contributions only on the $t b$ effective couplings, therefore in the numerical simulations we have taken

$$
\begin{aligned}
& d_{L}=\operatorname{diag}\left(K_{u d}, K_{c s}, g_{L}\right), \\
& d_{R}=\operatorname{diag}\left(0,0, g_{R}\right) .
\end{aligned}
$$

When we speak along the paper of the results for the standard model at the tree level we mean $g_{L}=1$, and $g_{R}=0$. However, even though numerical results are presented considering only the $t b$ entry $\left(g_{L}\right.$ and $\left.g_{R}\right)$, since flavor indices and masses are kept all along in the analytical expressions (see the Appendix), the appropriate changes to include other entries are immediate.

The effective couplings of the neutral sector (7) can be determined from the $Z \rightarrow f \bar{f}$ vertex $^{1}$ [12], but at present not much is known from the $t b$ effective coupling. This is perhaps best evidenced by the fact that the current experimental results for the (left-handed) $K_{t b}$ matrix element give [14]

$$
\frac{\left|K_{t b}\right|^{2}}{\left|K_{t d}\right|^{2}+\left|K_{t s}\right|^{2}+\left|K_{t b}\right|^{2}}=0.99 \pm 0.29
$$

In the standard model this matrix element is expected to be close to 1 . It should be emphasized that these are the "mea-

\footnotetext{
${ }^{1} \mathrm{~A} 3 \sigma$ discrepancy with respect to the standard model results, mostly due to the right-handed coupling, remains in the $Z$ couplings of the $b$ quark to this date.
} 
sured" or "effective" values of the CKM matrix elements, and that they do not necessarily correspond, even in the standard model, to the entries of a unitary matrix on account of the presence of radiative corrections. These deviations with respect to unitary are expected to be small-at the few percent level at most-unless new physics is present. At the Tevatron the left-handed couplings are expected to be eventually measured with a 5\% accuracy [15]. The present work is a contribution to such an analysis in the case of the LHC experiments.

As far as experimental bounds for the right handed effective couplings is concerned, the more stringent ones come at present from the measurements on the $b \rightarrow s \gamma$ decay at CLEO [16]. Due to a $m_{t} / m_{b}$ enhancement of the chirality flipping contribution, a particular combination of mixing angles and $\kappa_{R}^{C C}$ can be found. The authors of [17] reach the conclusion that $\left|\operatorname{Re}\left(\kappa_{R}^{C C}\right)\right| \leqslant 0.4 \times 10^{-2}$. However, considering $\kappa_{R}^{C C}$ as a matrix in generation space, this bound only constraints the $t b$ element. Other effective couplings involving the top quark remain virtually unrestricted from the data. The previous bound on the right-handed coupling is a very stringent one. It is pretty obvious that the LHC will not be able to compete with such a bound. Yet, the measurement will be a direct one, not through loop corrections. Equally important is that it will yield information on the $t d$ and $t s$ elements too, by just replacing the $\bar{b}$ quark in Figs. 1, 2 by a $\bar{d}$ or an $\bar{s}$, respectively.

Now we shall proceed to analyze the bounds that single top production at the LHC can set on the effective couplings. This combined with the data from $Z$ physics will allow an estimation of the six effective couplings (7), (8) in the matter sector of the effective electroweak Lagrangian. We will, in the present work limit ourselves to the consideration of the cross sections for production of polarized top quarks. We shall not consider at this stage the potential of measuring top decays angular distributions in order to establish relevant bounds on the effective electroweak couplings. This issue merits a more detailed analysis, including the possibility of detecting $C P$ violation [18].

\section{THE CROSS SECTION}

In order to calculate the cross section $\sigma$ of the process $p p \rightarrow t \bar{b}$ we have used the CTEQ4 set of structure functions [19] to determine the probability of extracting a parton with a given fraction of momenta from the proton. Hence we write schematically

$$
\sigma=\sum_{q} \int_{0}^{1} \int_{0}^{1} f_{g}(y) f_{q}(x) \hat{\sigma}\left(x P_{1}, y P_{2}\right) d x d y,
$$

where $f_{q}$ denote the parton distribution function (PDF) corresponding to the partonic quarks and antiquarks and $f_{g}$ indicate the PDF corresponding to the gluon. In Eq. (10) we have set the light quark and gluon momenta to $x P_{1}$ and $y P_{1}$, respectively. ( $P_{1}$ and $P_{2}$ are the four-momenta of the two colliding protons.) The approximation thus involves neglecting the transverse momenta of the incoming partons; the transverse fluctuations are integrated over by doing the appropriate integrals over $k_{T}$. We have then proceeded as follows. We have multiplied the parton distribution function of a gluon of a given momenta from the first proton by the sum of parton distribution functions for obtaining a $u$ type quark from the second proton. This result is then multiplied by the cross sections of the subprocesses of Fig. 1. We perform also the analogous process with the $\bar{d}$ type antiquarks of Fig. 2. At the end, these two partial results are add up to obtain the total $p p \rightarrow t \bar{b}$ cross section.

Typically the top quark decays weakly well before strong interactions become relevant, we can in principle measure its polarization state with virtually no contamination of strong interactions (see e.g. [20] for discussions this point and Sec. VI). For this reason we have considered polarized cross sections and provide general formulas for the production of polarized top quarks or top antiquarks. To this end one needs to introduce the spin projector

$$
\left(\frac{1+\gamma_{5} h h}{2}\right)
$$

with

$$
\begin{aligned}
& n^{\mu}=\frac{1}{\sqrt{\left(p_{1}^{0}\right)^{2}-\left(\vec{p}_{1} \cdot \hat{n}\right)^{2}}}\left(\vec{p}_{1} \cdot \hat{n}, p_{1}^{0} \hat{n}\right), \\
& \hat{n}^{2}=1, \quad n^{2}=-1,
\end{aligned}
$$

as the polarization projector for a particle or antiparticle of momentum $p_{1}$ with spin in the $\hat{n}$ direction. The calculation of the subprocesses cross sections have been performed for top quarks and top antiquarks polarized in an arbitrary direction $\hat{n}$. Later we have analyzed numerically different spin frames defined as follows.

Lab helicity frame: the polarization vector is taken in the direction of the three momentum of the top quark or top (right helicity) or in the opposite direction (left helicity).

Lab spectator frame: the polarization vector is taken in the direction of the three momentum of the spectator quark jet or in the opposite direction. The spectator quark is the $d$-type quark in Fig. 1 or the $\bar{u}$-type quark in Fig. 2.

Rest spectator frame: like in the Lab spectator frame we choose the spectator jet to define the polarization of the top quark or top antiquark. Here, however, we define $\hat{n}$ as \pm the direction of the three momentum of the spectator quark in the top quark or top antiquark rest frame (given by a pure boost transformation $\Lambda$ of the lab frame). Then we have $n_{r}$ $=(0, \hat{n})$ in that frame and $n=\Lambda^{-1} n_{r}$ back to the lab frame.

The calculation of the subprocess polarized cross section we present is completely analytical from beginning to end and the results are given in the Appendix. Both the kinematics and the polarization vector of the top quark (or top antiquark) are completely general. Since the calculation is of a certain complexity a number of checks have been done to ensure that no mistakes have been made. The integrated 
cross section agrees well with the results in [9] when the same cuts, scale, etc. are used. The mass of the top quark is obviously kept, but so is the bottom quark mass. The latter in fact turns out to be more relevant than expected as we shall see in a moment. As we have already discussed, the production of flavors other than $\bar{b}$ in association with the top quark can be easily derived from our results.

In single top production a distinction is often made between $2 \rightarrow 2$ and $2 \rightarrow 3$ processes. The latter corresponds, in fact, to the processes we have been discussing, the ones represented in Fig. 1, in which a gluon from the sea splits into a $b \bar{b}$ pair. In the $2 \rightarrow 2$ process the $b$ quark is assumed to be extracted from the sea of the proton, and both $b$ and $\bar{b}$ are collinear. Of course since the proton has no net $b$ content, a $\bar{b}$ quark must be present somewhere in the final state and the distinction between the two processes is purely kinematical. As is well known, when calculating the total cross section for single top production a logarithmic mass singularity [9] appears in the total cross section due to the collinear regime where the $b$ quark (and the $\bar{b}$ ) quark have $k_{T} \rightarrow 0$. This kinematic singularity is actually regulated by the mass of the bottom quark; it appears to all orders in perturbation theory and a proper treatment of this singularity requires the use of the Altarelli-Parisi equations and its resumation into a $b$ parton distribution function. While the evolution of the parton distribution functions is governed by perturbation theory, their initial values are not and some assumptions are unavoidable. Clearly an appropriate cut in $p_{T}$ should allow us to retain the perturbative regime of the $2 \rightarrow 3$ process, while suppressing the $2 \rightarrow 2$ one.

Two experimental approaches can be used at this point. One-advocated by Willenbrock and co-workers [9] -is to focus on the low $p_{T}$ regime. The idea is to minimize the contribution of the $t, \bar{t}$ background, whose characteristic angular distributions are more central. Then one is actually interested in processes where one does not see the $\bar{b}$ (respectively $b$ ) quark which is produced in association with the $t$ quark (respectively $\bar{t}$ ), and accordingly sets an upper cut on the $p_{T}$ of the $\bar{b}$. Clearly one then has to take into account the $2 \rightarrow 2$ process and, in particular, one must pay attention not to double count the low $p_{T}$ region [for the $\bar{b}$ (or $b$ ) quark] of the $2 \rightarrow 3$ process, which is already included via a $b$ PDF and has to be subtracted. This strategy has some risks. First of all, the separation between the $2 \rightarrow 3$ and $2 \rightarrow 2$ is not a clear cut one. The separation takes place in a region where the cross section is rapidly varying so the results do depend to some extend on the way the separation is done. Also as we just said relies on some initial condition for the $b$ PDF at some initial scale (for instance at $\mu=m_{b}$ ). Moreover, this strategy does not completely avoid the background originated in $\bar{t} t$ production either; for instance when in the decaying $\bar{t}$ $\rightarrow W^{-} \bar{b} \rightarrow \bar{u} d \bar{b}$ the $\bar{b}$ is missed along with the $\bar{u}$-type antiquark in which case the $d$-type quark is taken as the spectator or when the $\bar{b}$ is missed along with the $d$-type quark in which case the $\bar{u}$-type antiquark is taken as the spectator.
On the other hand, measuring the $\bar{b}$ (or $b$ for top antiquark production) momenta will allow a better kinematic reconstruction of the individual processes. This should allow for a separation from the dominant mechanism of top production through gluon fusion. Setting a sufficiently high upper cut for the jet energy and a good jet separation might be sufficient to avoid contamination from $t, \bar{t}$ when one hadronic jet is missed. Finally, the spin structure of the top quark is completely different in both cases due to the chiral couplings in electroweak production. Therefore, according to this philosophy we have implemented a lower cut of $30 \mathrm{GeV}$ in the transversal momentum of the $\bar{b}$ (respectively $b$ ) in top quark (respectively top antiquark) production.

We do not really want to make strong claims as to which strategy should prove more efficient eventually. Many different ingredients have to be taken into account. Just to mention one more: the results of our analysis show that the sensitivity to the right handed effective coupling is not very big and that the (subdominant) $s$-channel process may actually be more adequate for this purpose. Yet, this is again more central, so one will need to consider the $t$-channel process for largish values of $p_{T}$ anyway.

\section{A FIRST LOOK AT THE RESULTS}

We shall now present the results of our analysis. To calculate the total event production corresponding to different observables we have used the integrating Monte Carlo program VEGAS [21]. We present results after one year (defined as $10^{7}$ seg.) run at full luminosity in one detector $\left(100 \mathrm{fb}^{-1}\right.$ at $\mathrm{LHC}$ ).

The total contribution to the electroweak vertices $g_{L}, g_{R}$ has two sources: the effective operators parametrizing new physics, and the contribution from the universal radiative corrections. In the standard model, neglecting mixing, for example, we have a tree level contribution to the $\bar{t} W_{\mu}^{+} b$ vertex given by $-(i / \sqrt{2}) \gamma_{\mu} g K_{t b} L$. Radiative corrections (universal and $M_{H}$ dependent) modify $g_{L}$ and generate a nonzero $g_{R}$. These radiative corrections depend weakly on the energy of the process and thus in a first approximation we can take them as constant. Our purpose is to estimate the dependence of different LHC observables on these total effective couplings and how the experimental results can be used to set bounds on them. Assuming that the radiative corrections are known, this implies in turn a bound on the coefficients of the effective electroweak Lagrangian.

Let us start by discussing the experimental cuts. Because of the geometrical detector constraints we cut off very low angles for the outgoing particles. The top quark, bottom antiquark, and spectator quark have to come out with an angle in between 10 and 170 degrees. These angular cuts correspond to a cut in pseudorapidity $|\eta|<2$.44. In order to be able to detect the three jets corresponding to the outgoing particles we implements isolation cuts of 20 degrees between each other.

As already discussed we use a lower cut of $30 \mathrm{GeV}$ in the $\bar{b}$ jet. This reduces the cross section to less than one third of its total value, since typically the $\bar{b}$ quark comes out in the 


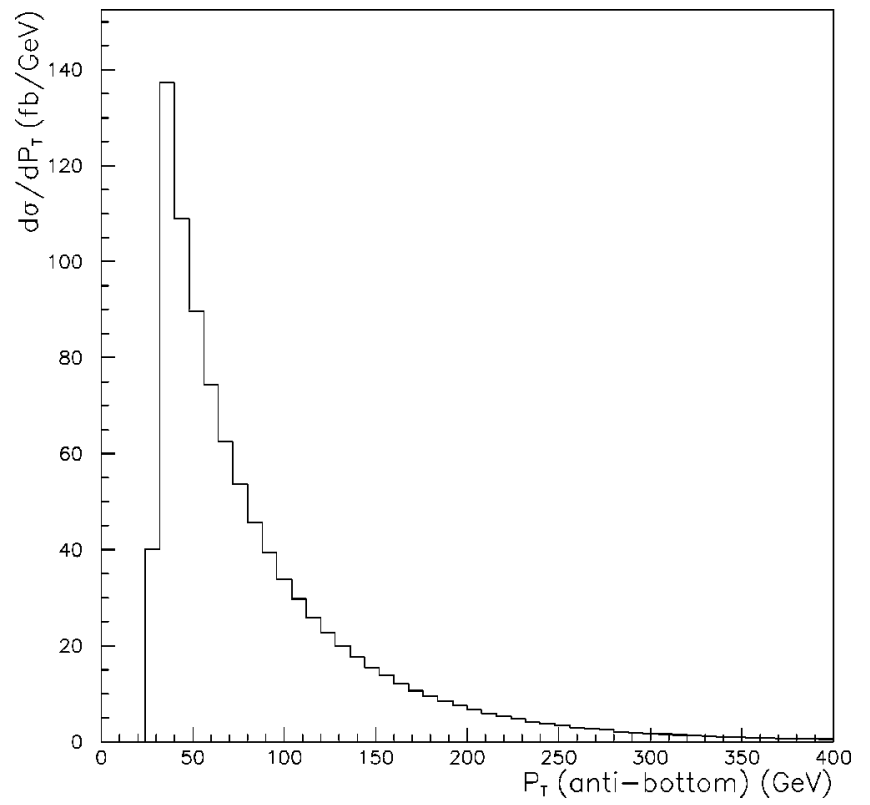

FIG. 3. Bottom antiquark transversal momentum distribution corresponding to unpolarized single top production at the LHC. The calculation was performed at the tree level in the standard model. Note the $30 \mathrm{GeV}$ cut implemented to avoid large logs due to the massless singularity in the total cross section. In this plot $\mu^{2}=\hat{s}$ $=\left(q_{1}+q_{2}\right)^{2}$.

same direction as the incoming gluon and a large fraction of them do not pass the cut (see Fig. 3). Similarly, $p_{T}>20 \mathrm{GeV}$ cuts are set for the top and spectator quark jets. These cuts guarantee the validity of perturbation theory and will serve to separate from the overwhelming background of low $p_{T}$ physics. These values come as a compromise to preserve a good signal, while suppressing unwanted contributions. They are similar, but not identical to the ones used in [9] and [11]. To summarize the allowed regions are

$$
\begin{gathered}
\text { detector geometry cuts: } 10^{\circ} \leqslant \theta_{i} \leqslant 170^{\circ}, \quad i=t, \bar{b}, q_{s}, \\
\text { isolation cuts: } 20^{\circ} \leqslant \theta_{i j}, \quad i, j=t, \bar{b}, q_{s},
\end{gathered}
$$

$$
\begin{gathered}
\text { theoretical cuts: } 20 \mathrm{GeV} \leqslant p_{1}^{T}, \quad 20 \mathrm{GeV} \leqslant q_{2}^{T}, \\
30 \mathrm{GeV} \leqslant p_{2}^{T},
\end{gathered}
$$

where $\theta_{t}, \theta_{\bar{b}}, \theta_{q_{s}}$ are the polar angles with respect to the beam line of the top quark, bottom antiquark and spectator quark respectively; $\theta_{t \bar{b}}, \theta_{t q_{s}}, \theta_{\bar{b} q_{s}}$ are the angles between top quark and bottom antiquark, top quark and spectator quark, and bottom antiquark and spectator quark, respectively. The momenta conventions are given in Figs. 1 and 2.

Numerically, the dominant contribution to the process comes from the diagram where a $b$ quark is exchanged in the $t$ channel, but a large amount of cancellation takes place with the crossed interference term with the diagram with a top quark in the $t$ channel. The smallest contribution (but obviously non-negligible) corresponds to this last diagram. It is then easy to see, given the relative smallness of the $b$ mass, why the process is so much forward.

Undoubtedly the largest theoretical uncertainty in the whole calculation is the choice of a scale for $\alpha_{s}$ and the PDF's. We perform a leading order calculation in QCD and the scale dependence is large. We have made two different choices. We present some results with the scale $p_{T}^{\text {cut }}$ used in $\alpha_{s}$ and the gluon PDF, while the virtuality of the $W$ boson is used as scale for the PDF of the light quarks in the proton. When we use these scales and compute, for instance, the total cross section above a cut of $p_{T}=20 \mathrm{GeV}$ in the $\bar{b}$ momentum, we get an excellent agreement with the calculations in [9]. Most of our results are however presented with a common scale $\mu^{2}=\hat{s}, \hat{s}$ being the center-of-mass energy squared of the $q g$ subprocess. The total cross section above the cut is then roughly speaking two thirds of the previous one, but no substantial change in the distributions takes place. It remains to be seen which one is the correct choice.

From our Monte Carlo simulation for single top quark production at the LHC after 1 year of full luminosity and with the cuts given above we obtain the total number of events. This number depends on the value of the effective couplings and on the top quark polarization vector $n$ given in the frames defined in Sec. III. If we call $N\left(g_{L}, g_{R}, \hat{n}\right.$, (frame) $)$ to this quantity, we obtain the following results:

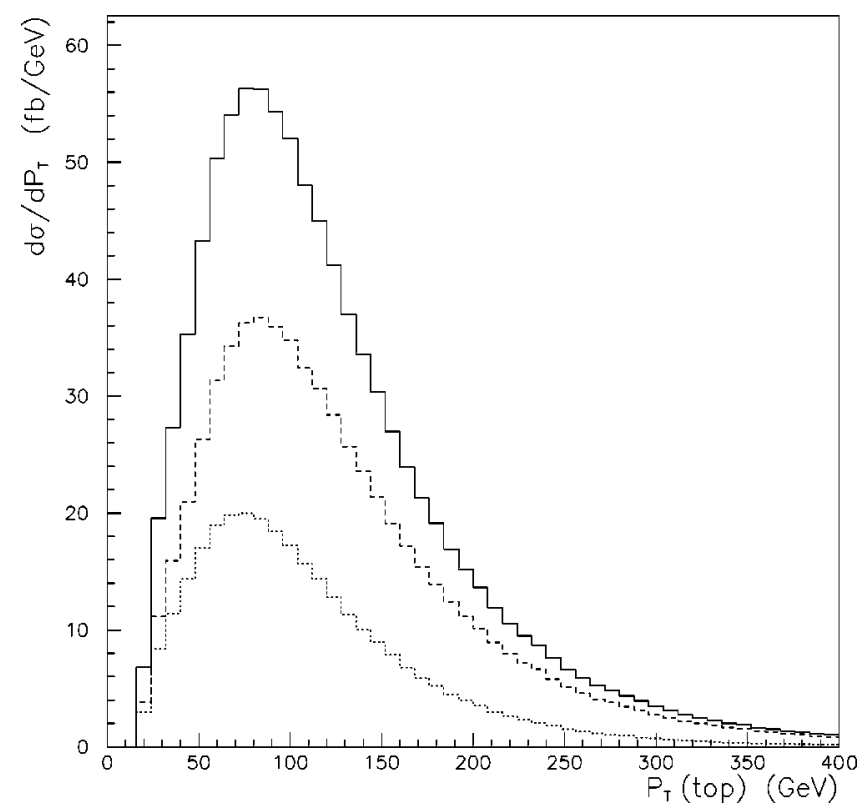

FIG. 4. Top transversal momentum distribution corresponding to polarized single top production at the LHC in the LAB system. The solid line corresponds to unpolarized top production and the dashed (dotted) line corresponds to top quarks of negative (positive) helicity. The subprocesses contributing to these histograms have been calculated at tree level in the electroweak theory. The cuts are described in the text. The degree of polarization in this spin basis and reference frame is only $69 \%$. The QCD scale is taken to be $\mu^{2}=\hat{s}=\left(q_{1}+q_{2}\right)^{2}$. 

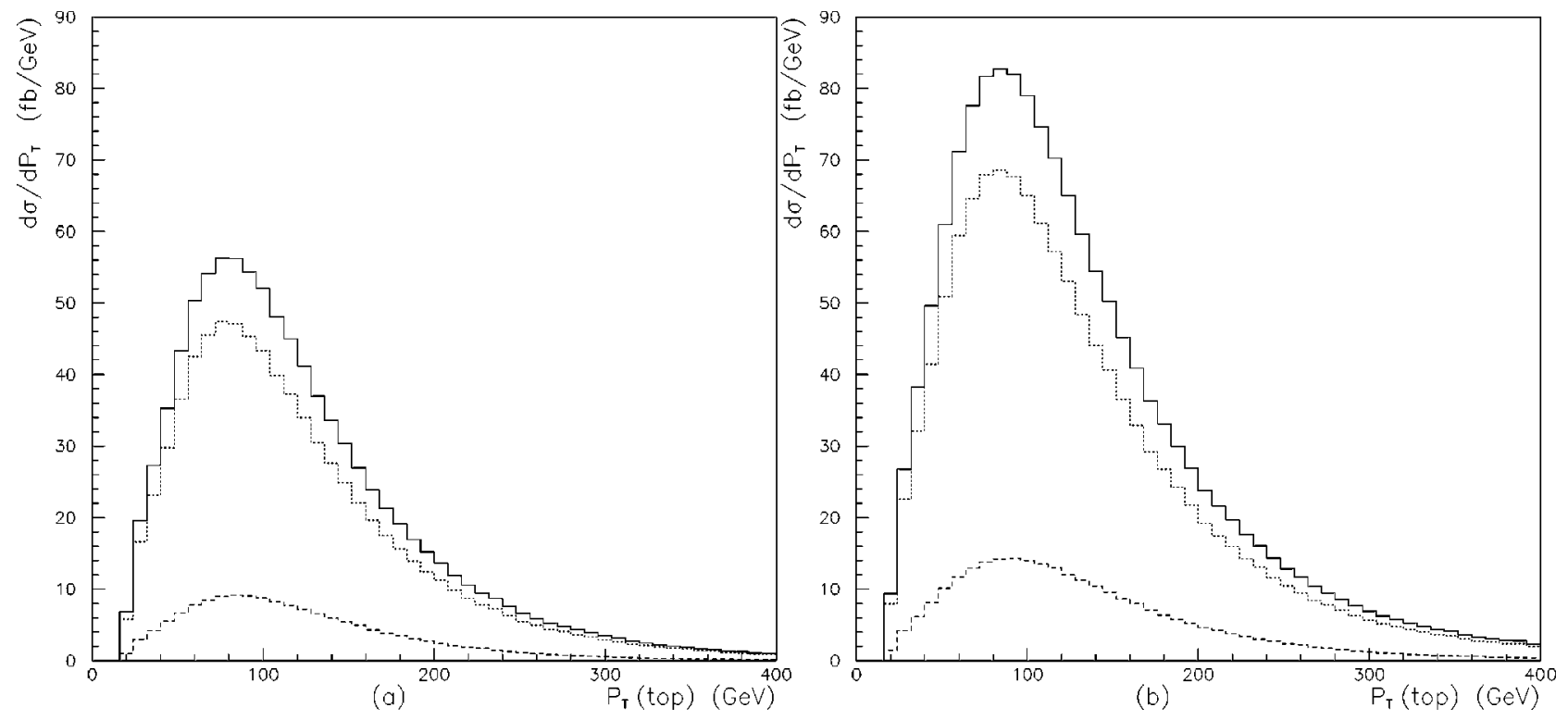

FIG. 5. Top transversal momentum distribution corresponding to polarized single top production at the LHC. The solid line corresponds to unpolarized top production and the dashed (dotted) line corresponds to top quarks polarized in the spectator jet negative (positive) direction in the top rest frame. In (a) the QCD scale is taken $\mu^{2}=\hat{s}=\left(q_{1}+q_{2}\right)^{2}$ and in (b) $\mu=p_{\text {cut }}^{T(\text { bot })}=30$ GeV. The subprocesses contributing to these histograms have been calculated at tree level in the electroweak theory. With our set of cuts, the polarization is in both cases $84 \%$.

$$
\begin{aligned}
& N\left(g_{L}, g_{R}, \hat{n}= \pm \frac{\vec{p}_{1}}{\left|\vec{p}_{1}\right|},(\mathrm{lab})\right) \\
& =g_{L}^{2} \times(3.73 \mp 1.31) \times 10^{5}+g_{R}^{2} \times(3.54 \pm 0.97) \times 10^{5} \\
& +g_{L} g_{R} \times(-0.237 \mp 0.0283) \times 10^{5} \text {, } \\
& N\left(g_{L}, g_{R}, \hat{n}= \pm \frac{\vec{q}_{2}}{\left|\vec{q}_{2}\right|},(\mathrm{lab})\right) \\
& =g_{L}^{2} \times(3.73 \pm 2.22) \times 10^{5}+g_{R}^{2} \times(3.54 \mp 2.12) \times 10^{5} \\
& +g_{L} g_{R} \times(-0.237 \mp 0.001) \times 10^{5}, \\
& N\left(g_{L}, g_{R}, \hat{n}= \pm \frac{\vec{q}_{2}}{\left|\vec{q}_{2}\right|},(\text { rest })\right) \\
& =g_{L}^{2} \times(3.73 \pm 2.49) \times 10^{5}+g_{R}^{2} \times(3.54 \mp 2.15) \times 10^{5} \\
& +g_{L} g_{R} \times(-0.237 \mp 0.0180) \times 10^{5} \text {, }
\end{aligned}
$$

where we have omitted the $O(\sqrt{N})$ statistical errors and we have neglected possible $C P$ phases $\left(g_{L}\right.$ and $g_{R}$ real). One can observe from the simulations that the production of negative helicity (left) top quarks represents the $69 \%$ of the total single top production (see Fig. 4), this predominance of left top quarks in the tree level electroweak approximation is expected due to the suppression at high energies of righthanded top quarks because of the zero right coupling in the charged current sector. In fact the production of right-handed top quarks would be zero were it not for the chirality flip,

due to the top quark mass, in the $t$-channel. Of course the name "left" and "right" are a bit misleading; we really mean negative and positive helicity states. Chirality states cannot be used, because the production is peaked in the 200 to 400

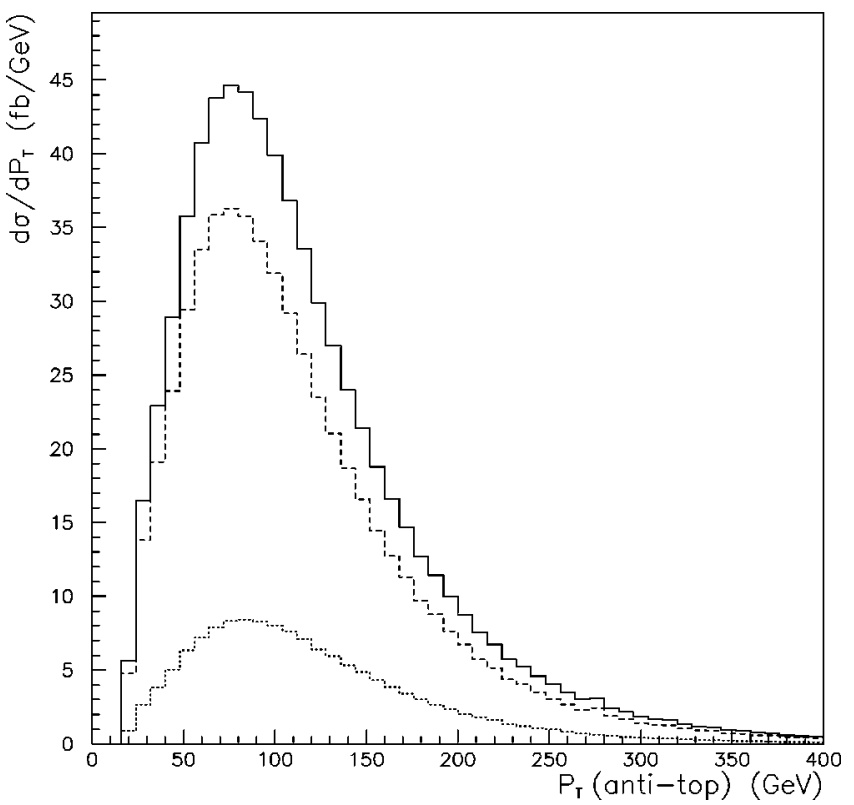

FIG. 6. Top antiquark transversal momentum distribution corresponding to polarized single top antiquark production at the LHC. The solid line corresponds to unpolarized top antiquark production and the dashed (dotted) line corresponds to top antiquarks polarized in the spectator jet negative (positive) direction in the top rest frame. The subprocesses contributing to these histograms have been calculated at the tree level in the electroweak theory, using the same cuts and conventions as in the previous figures. 


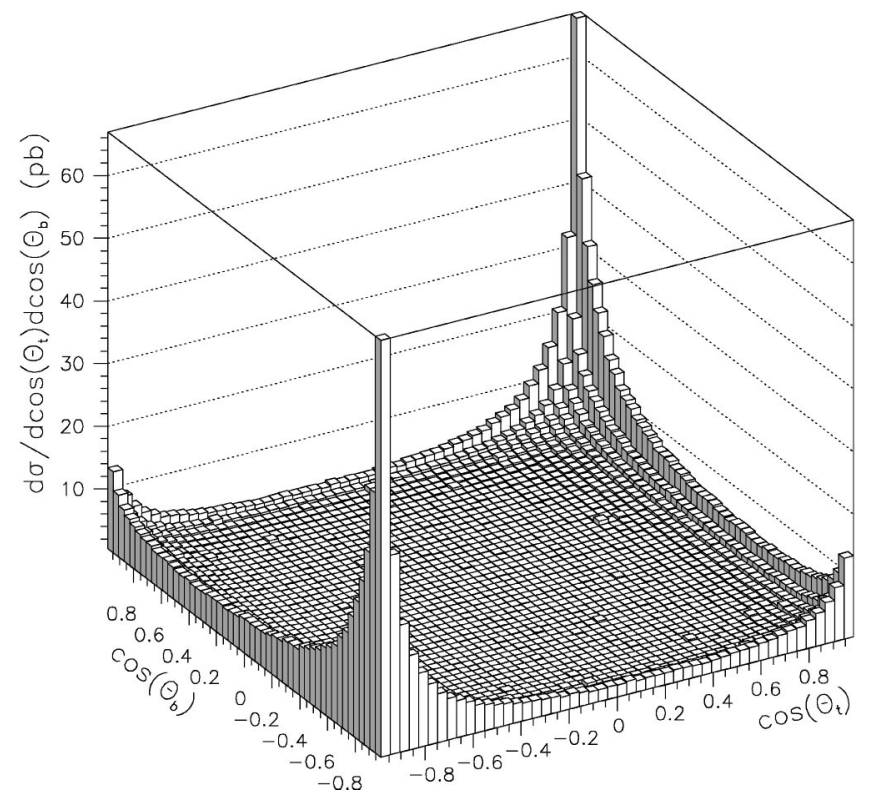

FIG. 7. Distribution of the cosines of the polar angles of the top and bottom antiquark with respect to the beam line. The plot corresponds to unpolarized single top production at the LHC. The calculation was performed at the tree level in standard model with $\mu^{2}$ $=\hat{s}=\left(q_{1}+q_{2}\right)^{2}$.

$\mathrm{GeV}$ region for the energy of the top quark and the mass cannot be neglected. The results for the production of tops polarized in the spectator jet direction in the top rest frame can be summarized in Fig. 5.

We have also calculated single top antiquark production obtaining a pattern similar to that of single top production

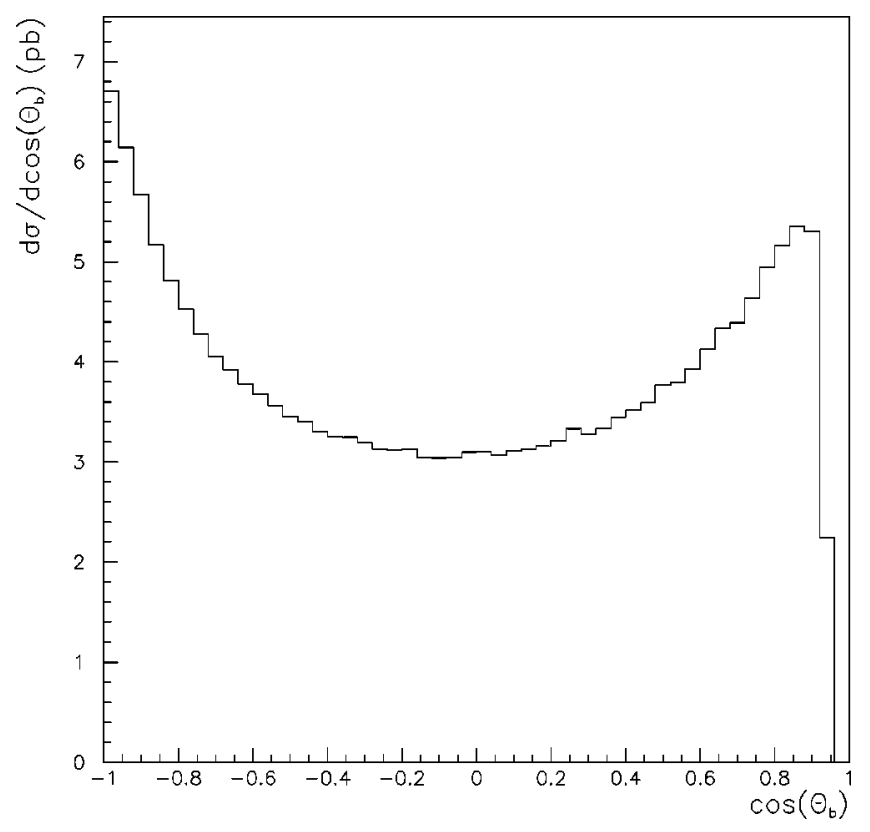

FIG. 8. Distribution of the cosine of the angle between top and bottom antiquark corresponding to unpolarized single top production at the LHC. The calculation was performed at the tree level in standard model with $\mu^{2}=\hat{s}=\left(q_{1}+q_{2}\right)^{2}$. The abrupt fall near 1 is due to the $20^{\circ}$ isolation cut.

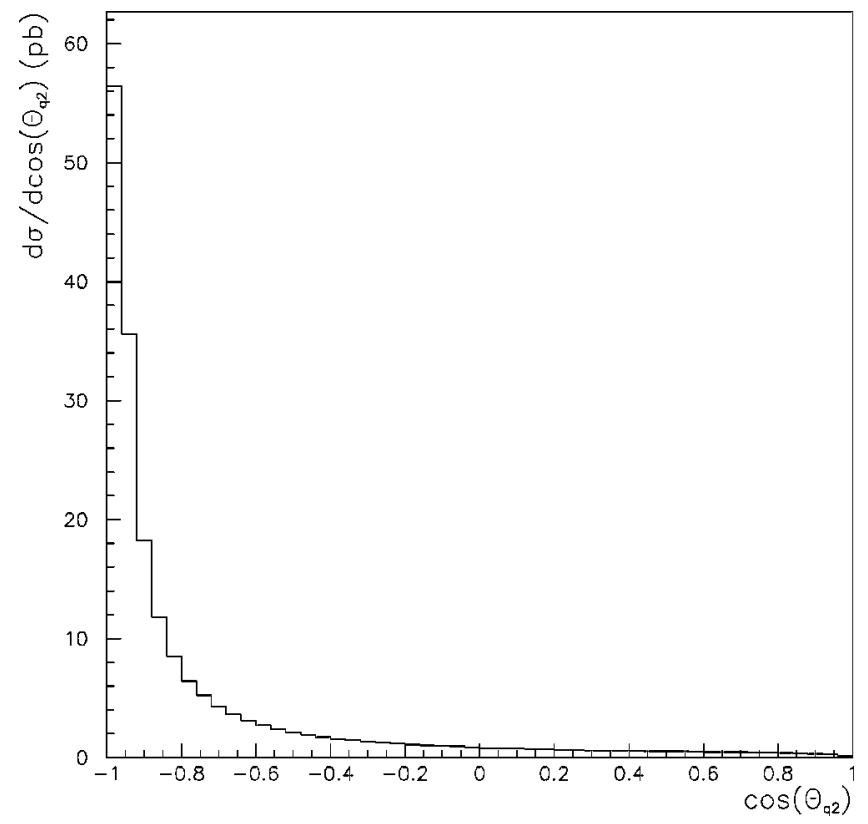

FIG. 9. Distribution of the cosine of the angle between the spectator quark and the gluon corresponding to unpolarized single top production at the LHC. The momentum of the gluon is in the beam line direction but its sense is not observable so to obtain an observable distribution we have to symmetrize the above one. The calculation was performed at the tree level in standard model with $\mu^{2}$ $=\hat{s}=\left(q_{1}+q_{2}\right)^{2}$.

but suppressed by an approximately $75 \%$ factor. This can be observed for example in Fig. 6. This suppression is generated by the parton distribution functions corresponding to negatively charged quarks that are smaller than the ones corresponding to positively charged quarks. Because of that the conclusions for top antiquark production are practically the same as the ones for top production taking into account such suppression and that, because of the transformations (A3) (see Appendix), passing from top quark to top antiquark is equivalent to changing the spin direction.

In Fig. 7 we plot the cross section distribution of the polar angles of the top quark and bottom antiquark with respect to the beam line for unpolarized single top production at the LHC. In Fig. 8 we plot the distribution of the cosine of the angle between the top quark and the bottom antiquark for unpolarized single top production at the LHC. Everything is calculated in the (tree level) standard model in the LAB frame. In both figures the above cuts are implemented, in particular the isolation cut of 20 degrees in the angle between the top quark and the bottom antiquark is clearly visible in Fig. 8. In Fig. 9 we also present the distribution of the cosine of the angle between the spectator quark and the gluon. From inspection of these figures two facts emerge: (a) the top-bottom distribution is strongly peaked in the beam direction as expected. (b) Even with the presence of the isolation cut, near the beam axis configurations with top quark and bottom antiquark almost parallel are favored with respect to back-to-back configurations. Therefore this is an indication that almost back-to-back configurations are distributed more uniformly in space than parallel configurations 

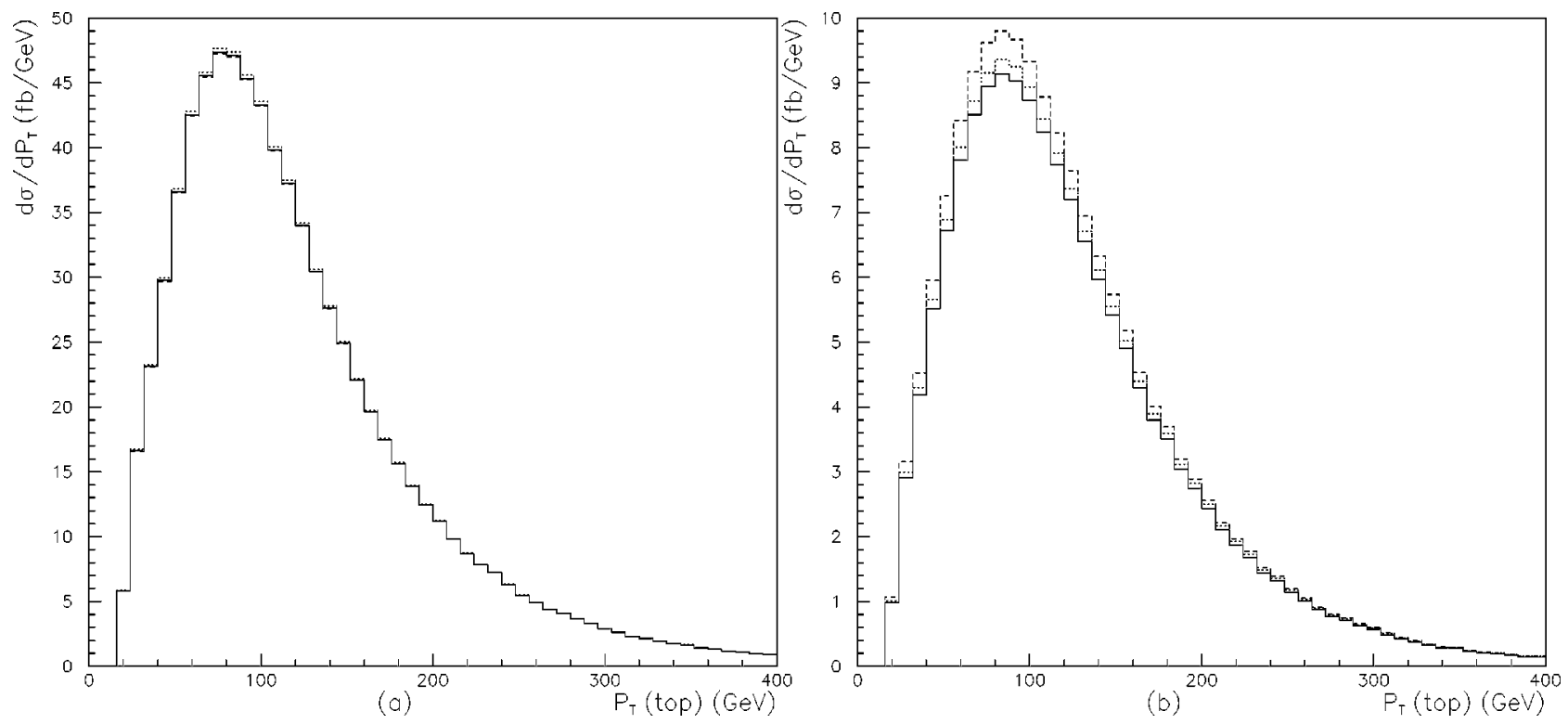

FIG. 10. Top transversal momentum distribution corresponding to polarized single top production at the LHC. Plots (a), (b) correspond to top quarks polarized in the spectator jet positive, negative direction respectively in the top rest frame. The subprocesses contributing to the solid line histogram have been calculated at the tree level in the SM $\left(g_{L}=1, g_{R}=0\right)$. The dashed (dotted) line histogram have been calculated at the tree level with $g_{L}=1$, and $g_{R}=0.1\left(g_{L}=1\right.$, and $\left.g_{R}=-0.1\right)$. Note in (a) that the variation in the cross section due to the variation of the right coupling around its SM tree level value is practically inappreciable.

favoring the beam line direction.

Let us now depart from the tree level standard model and consider nonzero values for $\delta g_{L}$ and $\delta g_{R}$. In what concerns the dependence on the right effective coupling, our results are summarized in Fig. 10. From that figure it is quite apparent that negatively polarized top quarks (in the top rest frame, as previously described) are more sensitive to the value of the right coupling.

Taking into account the results of Eq. (12) we can establish the intervals where the effective couplings are indistinguishable from their tree level standard model values taking a 1 sigma deviation as a rough statistical criterion. Evidently we do not pretend to make here a serious experimental analysis since we are not taking into account the full set of experimental and theoretical uncertainties. Our aim is just to present an order of magnitude estimate of the sensitivity of the different spin basis to the value of the effective coupling around their tree level standard model value. The results are given in Table I, where we indicate also the polarization vector chosen in each case. Of course those sensitivities (which, as said, are merely indicative) are calculated with the assumption that one could perfectly measure the top polarization in any of the above basis. As it is well known the top polarization is only measurable in an indirect way through the angular distribution of its decay products. In Sec. VI we outline the procedure to use our results to obtain a final angular distribution for the polarized top decay products (we believe that some confusion exists on this point). Obtaining that angular distribution involves a convolution of the single top production cross section with the decay products angular distribution and because of that we expect the true sensitivity to be worse than the ones given in Table I. Obviously such distribution is an observable quantity and therefore must be independent of the spin basis one uses at an intermediate step calculation (in other words, the results must be independent of the basis in which the top spin density matrix is written). Because of that the discussion as to which is the "best" basis for the top polarization is somewhat academic in our view.

TABLE I. Sensitivity of the polarized single top production to variations of the effective couplings. To calculate the intervals we have taken 2 sigma statistical deviations ( $95.5 \%$ confidence level) from tree level values as an order of magnitude criterion. Of course, given the uncertainties in the QCD scale, the overall normalization is dubious and the actual precision on $g_{L}$ a lot less. The purpose of these figures is to illustrate the relative accuracy. Between parenthesis we indicate the spin direction taken to calculate each interval.

\begin{tabular}{lcccc}
\hline \hline Polarization, frame & $g_{L}$ & $g_{R}$ & \\
$\hat{n}= \pm \frac{\vec{p}_{1}}{\left|\vec{p}_{1}\right|}$, lab & {$[0.9986,1.0014]$} & $(-)$ & {$[-0.26,0.85]$} & $(+)$ \\
$\hat{n}= \pm \frac{q_{2}}{\left|\vec{q}_{2}\right|}$, lab & {$[0.9987,1.0013]$} & $(+)$ & {$[-0.013,0.063]$} & $(-)$ \\
$\hat{n}= \pm \frac{q_{2}}{\left|\vec{q}_{2}\right|}$, rest & {$[0.9987,1.0013]$} & $(+)$ & {$[-0.021,0.059]$} & $(-)$ \\
\hline \hline
\end{tabular}


Any basis will do; if any, the natural basis is that one where the density matrix becomes diagonal, where production and decay factorize. This basis corresponds to none of the above. However it may still be useful to know that some basis are more sensitive to the effective couplings than others if one assumes (at least as a gedanken experiment) that the polarization of the top quark could be measured directly.

It is worth mentioning that the bottom quark mass, which appears in the cross section in crossed left-right terms, such as $m_{b} g_{L} g_{R}$, plays a crucial role in the actual determination of $g_{R}$. This is because from the $\left|\operatorname{Re}\left(\kappa_{R}^{C C}\right)\right| \leqslant 0.4 \times 10^{-2}$ bound [17] we expect $g_{L} g_{R} m_{b}>g_{R}^{2} m_{t}$. Evidently for the $t s$ or $t d$ couplings these terms are not expected to be so relevant.

\section{THE DIFFERENTIAL CROSS SECTION FOR POLARIZED TOP QUARKS}

We define the matrix elements of the subprocess of Figs. 1 and 2 as $M_{+}^{d}$ and $M_{+}^{\bar{u}}$, respectively. We also define the matrix elements corresponding to the processes producing top antiquarks as $M_{-}^{u}$, and $M_{-}^{\bar{d}}$. With these definitions the differential cross section for polarized tops $\sigma$ can be written schematically as

$$
A=c\left(\begin{array}{c}
f_{u}\left(a+a_{n}\right)+f_{\bar{d}}\left(b-b_{n}\right) \\
\frac{1}{2} f_{u}\left(c+c_{n}-i d_{n}\right)+\frac{1}{2} f_{\bar{d}}\left(c-c_{n}+i d_{n}\right)
\end{array}\right.
$$

and where $a, b, c, a_{n}, b_{n}, c_{n}$ and $d_{n}$ are independent of the effective couplings $g_{R}$ and $g_{L}$ and the subscripts $n$ indicate linear dependence on the top spin four-vector $n$. From Eq. (13) we observe that $A$ is an Hermitian matrix and therefore it is diagonalizable with real eigenvalues. Moreover, from the positivity of $\sigma$ we immediately arrive at the constraints

$$
\operatorname{det} A \geqslant 0 \text {, }
$$

$$
\operatorname{Tr} A \geqslant 0 \text {, }
$$

that is

$$
\begin{aligned}
& {\left[f_{u}\left(a+a_{n}\right)+f_{\bar{d}}\left(b-b_{n}\right)\right]\left[f_{u}\left(b+b_{n}\right)+f_{\bar{d}}\left(a-a_{n}\right)\right]} \\
& \quad \geqslant \frac{1}{4}\left(c^{2}\left(f_{u}+f_{\bar{d}}\right)^{2}+\left(c_{n}^{2}+d_{n}^{2}\right)\left(f_{u}-f_{\bar{d}}\right)^{2}+2 c c_{n}\left(f_{u}^{2}-f_{\bar{d}}^{2}\right)\right),
\end{aligned}
$$

and

$$
\sigma=c\left(f_{u}\left|M_{+}^{d}\right|^{2}+f_{\bar{d}}\left|M_{+}^{\bar{u}}\right|^{2}\right),
$$

where $f_{u}$ and $f_{\bar{d}}$ denote the parton distribution functions corresponding to extracting a $u$-type quark and a $\bar{d}$-type quark respectively and $c$ is a proportionality factor incorporating the kinematical and measure factors. Now using our analytical results for the matrix elements given in the Appendix along with Eq. (A3) and symmetries (A4) we obtain

$$
\begin{aligned}
\sigma= & c f_{u}\left[\left|g_{L}\right|^{2}\left(a+a_{n}\right)+\left|g_{R}\right|^{2}\left(b+b_{n}\right)+\frac{g_{R}^{*} g_{L}+g_{R} g_{L}^{*}}{2}\right. \\
& \left.\times\left(c+c_{n}\right)+i \frac{g_{L}^{*} g_{R}-g_{R}^{*} g_{L}}{2} d_{n}\right]+c f_{\bar{d}}\left[\left|g_{R}\right|^{2}\left(a-a_{n}\right)\right. \\
& +\left|g_{L}\right|^{2}\left(b-b_{n}\right)+\frac{g_{R}^{*} g_{L}+g_{R} g_{L}^{*}}{2}\left(c-c_{n}\right) \\
& \left.-i \frac{g_{L}^{*} g_{R}-g_{R}^{*} g_{L}}{2} d_{n}\right] \\
= & \left(g_{L}^{*} \quad g_{R}^{*}\right) A\left(\begin{array}{l}
g_{L} \\
g_{R}
\end{array}\right),
\end{aligned}
$$

where

$$
\left.\begin{array}{c}
\frac{1}{2} f_{u}\left(c+c_{n}+i d_{n}\right)+\frac{1}{2} f_{\bar{d}}\left(c-c_{n}-i d_{n}\right) \\
f_{u}\left(b+b_{n}\right)+f_{\bar{d}}\left(a-a_{n}\right)
\end{array}\right),
$$

$$
\left(f_{u}+f_{\bar{d}}\right)(a+b)+\left(f_{u}-f_{\bar{d}}\right)\left(a_{n}+b_{n}\right) \geqslant 0 .
$$

Note that it is not possible to saturate both constraints for the same configuration because this would imply a vanishing $A$ which in turn would imply relations such as

$$
\frac{a+b}{a_{n}+b_{n}}=\frac{f_{\bar{d}}-f_{u}}{f_{\bar{d}}+f_{u}}=\frac{a_{n}-b_{n}}{a-b},
$$

which evidently do not hold. Moreover, since constraints (16) and (17) must be satisfied for any set of positive PDF's we immediately obtain the bounds

$$
\begin{gathered}
a b+a_{n} b_{n}-\frac{1}{4}\left(c^{2}+c_{n}^{2}+d_{n}^{2}\right) \geqslant\left|a_{n} b+a b_{n}-\frac{1}{2} c c_{n}\right| \\
b^{2}+a^{2}-\left(b_{n}^{2}+a_{n}^{2}\right) \geqslant \frac{1}{2}\left(c^{2}-\left(c_{n}^{2}+d_{n}^{2}\right)\right) .
\end{gathered}
$$

In order to have a $100 \%$ polarized top we need a spin fourvector $n$ that saturates the constraint (14) [that is Eq. (16)] for 
each kinematical situation, that is we need $A(n)$ to have a zero eigenvalue which is equivalent to have a unitary matrix $C$ satisfying

$$
C^{\dagger} A C=\operatorname{diag}(\lambda, 0),
$$

for some positive eigenvalue $\lambda$. In general such $n$ need not exist and, should it exist, is in any case independent of the effective couplings $g_{R}$ and $g_{L}$. Moreover, provided this $n$ exists there is only one solution (up to a global complex normalization factor $\alpha$ ) for the pair $\left(g_{R}, g_{L}\right)$ to the equation $\sigma=0$. This solution is just

$$
\begin{aligned}
& g_{L}=\alpha C_{12}, \\
& g_{R}=\alpha C_{22} .
\end{aligned}
$$

Note that if one of the effective couplings vanishes we can take the other constant and arbitrary. However if both effective couplings are nonvanishing we would have a quotient $g_{R} / g_{L}$ that would depend in general on the kinematics. This is not possible so we can conclude that for a nonvanishing $g_{R}$ ( $g_{L}$ is evidently nonvanishing) it is not possible to have a pure spin state (or, else, only for fine tuned $g_{R}$ a $100 \%$ polarization is possible).

To illustrate these considerations let us give an example: in the unphysical situation where $m_{t} \rightarrow 0$ it can be shown that there exists two solutions to the saturated constraint (14), namely

$$
m_{t} n^{\mu} \rightarrow \pm\left(\left|\vec{p}_{1}\right|, p_{1}^{0} \frac{\vec{p}_{1}}{\left|\vec{p}_{1}\right|}\right)
$$

once we have found this result we plug it in the expression (18) and we find the solutions $\left(0, g_{L}\right)$ with $g_{L}$ arbitrary for the $+\operatorname{sign}$ and $\left(g_{R}, 0\right)$ with $g_{R}$ arbitrary for the - sign. That is, physically we have zero probability of producing a right handed top quark when we have only a left handed coupling and vice versa when we have only a right handed coupling. Note that in this case it is clear that having both effective couplings nonvanishing would imply the absence of $100 \%$ polarization in any spin basis. This can be understood in general remembering that the top particle forms in general an entangled state with the other particles of the process. Since we are tracing over the unknown spin degrees of freedom and over the flavors of the spectator quark we do not expect in general to end up with a top quark in a pure polarized state; although this is not impossible as it is shown the in the last example.

In the physical situation where $m_{t} \neq 0$ (we use $m_{t}$ $=175.6 \mathrm{GeV}$ and $m_{b}=5 \mathrm{GeV}$ in this paper) we have found that a spin basis with relatively high polarization is the one with the spin $\hat{n}$ taken in the direction of the spectator quark in the top rest frame. This is in accordance to the results in [11]. In general the degree of polarization $\left[N_{+} \hat{n} /\left(N_{+} \hat{n}\right.\right.$ $\left.\left.+N_{-} \hat{n}\right)\right]$ depends not only on the spin frame but also on the particular cuts chosen. We have found that the lower cut for the transverse momentum of the bottom quark worsens the polarization degree but, in spite of that, from Eq. (12) we see that we have a $84 \%$ of polarization in the standard model $\left(g_{L}=1, g_{R}=0\right)$ that is much bigger than the $69 \%$ obtained with the helicity frame. The above results follow the general trend of those presented by Mahlon and Parke [11], but in general, their degree of polarization is higher. We understand that this is due to the different cuts (in particular for the transversal momentum of the bottom) along with the different set of parton distribution functions (PDF's) used in our simulations.

\section{MEASURING THE TOP POLARIZATION FROM ITS DECAY PRODUCTS}

A well known result in the tree level SM regarding the measure of the top polarization from its decay products is the formula that states the following: Given a top quark polarized in the $\hat{n}$ direction in its rest frame, the lepton $l^{+}$produced in the decay of the top quark via the process

$$
t \rightarrow b\left(W^{+} \rightarrow l^{+} \nu_{l}\right)
$$

presents an angular distribution [22]

$$
\sigma_{l}=\alpha(1+\cos \theta)
$$

where $\alpha$ is a normalization factor and $\theta$ is the axial angle measured from the direction of $\hat{n}$. What can we do when the top quark is in a mixed state with no $100 \%$ polarization in any direction? The first naive answer would be: With any axis $\hat{n}$ in the top rest frame the top quark will have a polarization $p_{+}$(with $0 \leqslant p_{+} \leqslant 1$ ) in that direction and a polarization $p_{-}=1-p_{+}$in the opposite direction so the angular distribution for the lepton is

$$
\begin{aligned}
\sigma_{l} & =\alpha\left[p_{+}(1+\cos \theta)+p_{-}(1-\cos \theta)\right] \\
& =\alpha\left[1+\left(p_{+}-p_{-}\right) \cos \theta\right] \\
& =\alpha\left[1+\left(2 p_{+}-1\right) \cos \theta\right]
\end{aligned}
$$

The problem with formula (22) is that the angular distribution for the lepton depends on the arbitrary chosen axis $\hat{n}$ and this cannot be correct. The correct answer can be obtained by noting the following facts:

Given an arbitrary chosen axis $\hat{n}$ in the rest frame and the associated spin basis to it $\{|+\hat{n}\rangle,|-\hat{n}\rangle\}$ the top spin state in given by a $2 \times 2$ density matrix $\rho$

$$
\begin{aligned}
\rho= & \rho_{+}|+\hat{n}\rangle\left\langle+\hat{n}\left|+\rho_{-}\right|-\hat{n}\right\rangle\langle-\hat{n}|+b|+\hat{n}\rangle\langle-\hat{n}| \\
& +b^{*}|-\hat{n}\rangle\langle+\hat{n}|
\end{aligned}
$$

which is in general not diagonal $(b \neq 0)$ and whose coefficients depend on the rest of kinematical variables determining the differential cross section. 
From the calculation of the polarized cross section we only know the diagonal elements $\rho_{ \pm}=p_{ \pm}=|M|_{ \pm \hat{n}}^{2} /\left(|M|_{+\hat{n}}^{2}\right.$ $\left.+|M|_{-\hat{n}}^{2}\right)$.

Given $\rho$ in any orthogonal basis determined (up to phases) by $\hat{n}$ we can change to another basis that diagonalizes $\rho$. Since the top quark is a spin-1/2 particle, this basis will correspond to another direction $\hat{n}_{d}$.

Once we have $\rho$ diagonalized then Eq. (22) is trivially correct with $p_{ \pm}=\rho_{ \pm}$and now $\theta$ is unambiguously measured from the direction of $\hat{n}_{d}$.

From the above facts the first question that comes to our minds is if there exists a way to determine $\hat{n}_{d}$ without knowing the off-diagonal matrix elements of $\rho$. The answer is yes. It is an easy exercise of elementary quantum mechanics that given a $2 \times 2$ Hermitian matrix $\rho$ the eigenvector with largest (lowest) eigenvalue correspond to the unitary vector that maximizes (minimizes) the bilinear form $\langle v|\rho| v\rangle$ constrained to $\{|v\rangle,\langle v \mid v\rangle=1\}$. Since an arbitrary normalized $|v\rangle$ can be written (up to phases) as $|+\hat{n}\rangle$ and in that case $\rho_{+}=p_{+}$then the correct $\hat{n}_{d}$ entering in Eq. (22) is the one that maximizes the differential cross section $|M|_{\hat{n}}^{2}$ for each kinematical configuration. At the end, the correct angular distribution for the leptons is given by the cross section for polarized top quarks in this basis $\left(\hat{n}_{d}\right)$ convoluted with formula (21) (or improvements of it [23]).

The above analysis was carried out in the standard model $\left(g_{R}=0\right)$ but it is correct also for $g_{R} \neq 0$ using the complete formula for this case

$$
\sigma_{l}=\alpha\left(1+\left(p_{+}-p_{-}\right) \cos \theta\left[1-\frac{1}{4}\left|g_{R}\right|^{2} h\left(\frac{M_{W}^{2}}{m_{t}^{2}}\right)\right]\right),
$$

where $h\left(M_{W}^{2} / m_{t}^{2}\right) \simeq 0.566$ [22]. Formula (24) deserves some comments:

First of all we remember that $\theta$ is the angle (in the top rest frame) between the $\hat{n}$ that maximizes the difference $\left(p_{+}\right.$ $\left.-p_{-}\right)$and the three momentum of the lepton.

Taking into account the above comment and that $\left(p_{+}\right.$ $-p_{-}$) depends on $g_{L}$ and $g_{R}$ we see that also $\theta$ depends on $g_{L}$ and $g_{R}$.

From the computational point of view, formula (24) is not an explicit formula because involves a process of maximization for each kinematical configuration.

In some works in the literature [11] formula (24) is presented for an arbitrary choice of the spin basis $\{| \pm \hat{n}\rangle\}$ in the top rest frame. This is incorrect because it does not take into account that, in general, the top spin density matrix is not diagonal.

In a recent work [23] $O\left(\alpha_{s}\right)$ corrections are incorporated to the polarized top decay angular analysis. In this work the density matrix for the top spin is properly taken into account. To connect this work with ours we have to replace their polarization vector $\vec{P}$ by $P \hat{n}_{d}$ where the magnitude of the top polarization $P$ is just the spin asymmetry $\left(|M|_{+\hat{n}_{d}}^{2}\right.$
$\left.-|M|_{-\hat{n}_{d}}^{2}\right) /\left(|M|_{+\hat{n}_{d}}^{2}+|M|_{-\hat{n}_{d}}^{2}\right)$ in our language. This taken into account, the density matrix the authors of [23] quote is in the basis $\left\{\left|+\hat{n}_{W^{+}}\right\rangle,\left|-\hat{n}_{W^{+}}\right\rangle\right\}$where $\hat{n}_{W^{+}}$is a normal vector in the direction of the three momentum of the $W^{+}$(in the top rest frame).

\section{CONCLUSIONS}

We have done a complete calculation of the subprocess cross sections for polarized top quarks or top antiquarks including the right effective coupling and bottom quark mass corrections. We have used a $p_{T}>30 \mathrm{GeV}$ cut in the transverse momentum of the produced $\bar{b}$ quark and, accordingly we have retained only the so called $2 \rightarrow 3$ process, for the reasons described in the text.

Our analysis here is completely general. No approximation is made. We use the most general set of couplings and, since our approach is completely analytical, we can describe the contribution from other intermediate quarks in the $t$ channel, mixing, etc. Masses and mixing angles are retained. On the contrary, the analysis has to be considered only preliminary from an experimental point of view. No detailed study of the backgrounds has been made, except for the dominant $g g \rightarrow t \bar{t}$ process which has been considered to some extent (although again without quantitative evaluation).

Given the (presumed) smallness of the right handed couplings, the bottom quark mass plays a role which is more important than anticipated, as the mixed crossed $g_{L} g_{R}$ term, which actually is the most sensitive one to $g_{R}$ is accompanied by a $b$ quark mass. The statistical sensitivity to different values of this coupling is given in the text.

We present a variety of $p_{T}$ and angular distributions both for the $t$ and the $\bar{b}$ quarks. Obviously, the top decays shortly after production, but we have not made detailed simulations of this part. In fact, the interest of this decay is obvious: one can measure the spin of the top quark through the angular distribution of the leptons produced in this decay. In the standard model, single top quark production gives a high degree of polarization ( $84 \%$ in the optimal basis, with the present set of cuts). This is a high degree of polarization, but well below the 90+ claimed by Mahlon and Parke in [11]. We understand this being due to the presence of the $30 \mathrm{GeV}$ cut. In fact, if we remove this cut completely we get a $91 \%$ polarization. Still below the result of [11] but in rough agreement (note that we do not include the $2 \rightarrow 2$ process). Inasmuch as they can be compared our results are in good agreement with those presented in [9] in what concerns the total cross section. Two different choices for the strong scale $\mu^{2}$ are presented.

In addition, it turns out that when $g_{R} \neq 0$ the top quark can never be $100 \%$ polarized. In other words, it is in a mixed state. In this case we show that a unique spin basis is singled out which allows one to connect top decay products angular distribution with the polarized top differential cross section.

Finally it should be mentioned that a previous study for this process in the present context was performed by the present authors [24] using the effective $\mathrm{W}$ approximation [25], in which the $W$ is treated as a parton of the proton. 
While this is certainly not an exact treatment, it is expected to be sufficiently good for our purposes. In the course of this work we have found, however, a number of interesting differences.

\section{ACKNOWLEDGMENTS}

We would like to thank A. Dobado, S. Forte, M.J. Herrero, J.R. Peláez, and E.Ruíz-Morales for multiple discussions. We would also like to thank W. Hollik for encouragement and for allowing us to present preliminary results in the LHC CERN Workshop. J.M. acknowledges financial support from Generalitat de Catalunya, grant 1998FI-00614. Financial support from grants AEN98-0431, 1998SGR 00026 and EURODAPHNE is greatly appreciated.

\section{APPENDIX: SUBPROCESSES CROSS SECTIONS}

In this appendix we present the analytical results obtained for the matrix elements $M_{+}^{d}$ and $M_{+}^{\bar{u}}$ corresponding to the processes of Figs. 1 and 2 respectively and the ones corresponding to top antiquark production $M_{-}^{u}$ and $M_{-}^{\bar{d}}$. Defining

$$
\begin{aligned}
& g_{+}=g_{R}, \\
& g_{-}=g_{L},
\end{aligned}
$$

we have the square modulus

$$
\begin{aligned}
\left|M_{-}^{u}\right|^{2}= & g_{s}^{2}\left(O_{11} A_{11}+O_{22} A_{22}+O_{c}\left(A_{p}^{(+)}+A_{p}^{(-)}+A_{m_{t}}^{(+)}+A_{m_{t}}^{(-)}\right.\right. \\
& \left.\left.+A_{m_{b}}^{(+)}+A_{m_{b}}^{(-)}\right)\right),
\end{aligned}
$$

with

$$
\begin{aligned}
O_{11} & =\frac{1}{4\left(k_{1} \cdot p_{1}\right)^{2}}, \\
O_{22} & =\frac{1}{4\left(k_{1} \cdot p_{2}\right)^{2}}, \\
O_{c} & =\frac{1}{4\left(k_{1} \cdot p_{1}\right)\left(k_{1} \cdot p_{2}\right)},
\end{aligned}
$$

and

$$
\begin{aligned}
A_{11}= & \frac{|g|^{4}\left|K_{u d}\right|^{2}}{\left(k_{2}^{2}-M_{W}^{2}\right)^{2}}\left\{i m_{t}^{2} m_{b} \frac{g_{L}^{*} g_{R}-g_{R}^{*} g_{L}}{2} \varepsilon^{\mu \nu \alpha \beta}\left(k_{1}-p_{1}\right)_{\mu} n_{\nu} q_{2 \alpha} q_{1 \beta}+m_{t} m_{b} \frac{g_{R}^{*} g_{L}+g_{L}^{*} g_{R}}{2}\left[m_{t}\left(q_{2} \cdot\left(k_{1}-p_{1}\right)\right)\left(q_{1} \cdot n\right)\right.\right. \\
& \left.-m_{t}\left(q_{1} \cdot\left(k_{1}-p_{1}\right)\right)\left(q_{2} \cdot n\right)-\left(q_{1} \cdot q_{2}\right)\left(m_{t}^{2}-\left(k_{1} \cdot p_{1}\right)\right)\right]+2\left|g_{L}\right|^{2}\left(q_{2} \cdot p_{2}\right)\left[\left(m_{t}^{2}+\frac{p_{1}+m_{t} n}{2} \cdot\left(k_{1}-p_{1}\right)\right)\left(q_{1} \cdot\left(k_{1}-p_{1}\right)\right)\right. \\
& \left.-\frac{1}{2} m_{t}^{3}\left(n \cdot q_{1}\right)+\left(\frac{p_{1}+m_{t} n}{2} \cdot q_{1}\right)\left(k_{1} \cdot p_{1}\right)\right]+2\left|g_{R}\right|^{2}\left(q_{1} \cdot p_{2}\right)\left[\left(m_{t}^{2}+\frac{p_{1}-m_{t} n}{2} \cdot\left(k_{1}-p_{1}\right)\right)\left(q_{2} \cdot\left(k_{1}-p_{1}\right)\right)+\frac{1}{2} m_{t}^{3}\left(n \cdot q_{2}\right)\right. \\
& \left.\left.+\left(\frac{p_{1}-m_{t} n}{2} \cdot q_{2}\right)\left(k_{1} \cdot p_{1}\right)\right]\right\},
\end{aligned}
$$

and

$$
\begin{aligned}
A_{22}= & \frac{|g|^{4}\left|K_{u d}\right|^{2}}{\left(k_{2}^{2}-M_{W}^{2}\right)^{2}}\left\{\left(k_{1} \cdot p_{2}\right)\left[2\left|g_{R}\right|^{2}\left(q_{1} \cdot k_{1}\right)\left(q_{2} \cdot \frac{p_{1}-m_{t} n}{2}\right)+2\left|g_{L}\right|^{2}\left(q_{2} \cdot k_{1}\right)\left(q_{1} \cdot \frac{p_{1}+m_{t} n}{2}\right)\right]+m_{b}^{2}\left[2\left|g_{R}\right|^{2}\left(q_{1} \cdot\left(k_{1}-p_{2}\right)\right)\right.\right. \\
& \left.\times\left(q_{2} \cdot \frac{p_{1}-m_{t} n}{2}\right)+2\left|g_{L}\right|^{2}\left(q_{2} \cdot\left(k_{1}-p_{2}\right)\right)\left(q_{1} \cdot \frac{p_{1}+m_{t} n}{2}\right)\right]+m_{b} \frac{g_{L}^{*} g_{R}+g_{R}^{*} g_{L}}{2}\left(m_{b}^{2}-\left(k_{1} \cdot p_{2}\right)\right)\left[-m_{t}\left(q_{1} \cdot q_{2}\right)-\left(q_{1} \cdot n\right)\right. \\
& \left.\left.\times\left(q_{2} \cdot p_{1}\right)+\left(q_{2} \cdot n\right)\left(q_{1} \cdot p_{1}\right)\right]-i m_{b} \frac{g_{L}^{*} g_{R}-g_{R}^{*} g_{L}}{2}\left(m_{b}^{2}-\left(k_{1} \cdot p_{2}\right)\right) \varepsilon^{\mu \nu \alpha \beta} n_{\mu} p_{1 \nu} q_{2 \alpha} q_{1 \beta}\right\},
\end{aligned}
$$

and

$$
\begin{aligned}
A_{p}^{( \pm)}= & -\frac{|g|^{4}\left|K_{u d}\right|^{2}}{\left(k_{2}^{2}-M_{W}^{2}\right)^{2}}\left|g_{ \pm}\right|^{2}\left\{( q _ { 1 } \cdot q _ { 2 } ) \left[\left(\left(k_{1}-p_{1}\right) \cdot\left(k_{2}-p_{1}\right)\right)\left(\frac{p_{1} \mp m_{t} n}{2} \cdot p_{2}\right)+\left(\left(k_{1}-p_{1}\right) \cdot \frac{p_{1} \mp m_{t} n}{2}\right)\left(\left(k_{2}-p_{1}\right) \cdot p_{2}\right)\right.\right. \\
& \left.-\left(\left(k_{1}-p_{1}\right) \cdot p_{2}\right)\left(\frac{p_{1} \mp m_{t} n}{2} \cdot\left(k_{2}-p_{1}\right)\right)\right]+\left(\left(k_{2}-p_{1}\right) \cdot q_{2}\right)\left[\left(p_{2} \cdot\left(k_{1}-p_{1}\right)\right)\left(q_{1} \cdot \frac{p_{1} \mp m_{t} n}{2}\right)\right. \\
& \left.-\left(q_{1} \cdot p_{2}\right)\left(\left(k_{1}-p_{1}\right) \cdot \frac{p_{1} \mp m_{t} n}{2}\right)\right]-\left(\left(k_{1}-p_{1}\right) \cdot q_{2}\right)\left[\left(p_{2} \cdot\left(k_{2}-p_{1}\right)\right)\left(q_{1} \cdot \frac{p_{1} \mp m_{t} n}{2}\right)-\left(q_{1} \cdot p_{2}\right)\left(\left(k_{2}-p_{1}\right) \cdot \frac{p_{1} \mp m_{t} n}{2}\right)\right]
\end{aligned}
$$




$$
\begin{aligned}
& +\left(\left(k_{2}-p_{1}\right) \cdot q_{1}\right)\left[\left(p_{2} \cdot\left(k_{1}-p_{1}\right)\right)\left(q_{2} \cdot \frac{p_{1} \mp m_{t} n}{2}\right)-\left(q_{2} \cdot p_{2}\right)\left(\left(k_{1}-p_{1}\right) \cdot \frac{p_{1} \mp m_{t} n}{2}\right)\right]-\left(\left(k_{1}-p_{1}\right) \cdot q_{1}\right)\left[\left(p_{2} \cdot\left(k_{2}-p_{1}\right)\right)\right. \\
& \left.\times\left(q_{2} \cdot \frac{p_{1} \mp m_{t} n}{2}\right)-\left(q_{2} \cdot p_{2}\right)\left(\left(k_{2}-p_{1}\right) \cdot \frac{p_{1} \mp m_{t} n}{2}\right)\right] \pm\left(\left(k_{1}-p_{1}\right) \cdot\left(k_{2}-p_{1}\right)\right)\left[\left(\left(\frac{p_{1} \mp m_{t} n}{2}\right) \cdot q_{2}\right)\left(p_{2} \cdot q_{1}\right)\right. \\
& \left.\left.-\left(\left(\frac{p_{1} \mp m_{t} n}{2}\right) \cdot q_{1}\right)\left(p_{2} \cdot q_{2}\right)\right] \pm\left(\frac{p_{1} \mp m_{t} n}{2} \cdot p_{2}\right)\left[\left(\left(k_{1}-p_{1}\right) \cdot q_{2}\right)\left(\left(k_{2}-p_{1}\right) \cdot q_{1}\right)-\left(\left(k_{1}-p_{1}\right) \cdot q_{1}\right)\left(\left(k_{2}-p_{1}\right) \cdot q_{2}\right)\right]\right\},
\end{aligned}
$$

and

$$
\begin{aligned}
A_{m_{t}}^{( \pm)}= & \frac{|g|^{4}\left|K_{u d}\right|^{2}}{\left(k_{2}^{2}-M_{W}^{2}\right)^{2}} \frac{\left|g_{ \pm}\right|^{2}}{2}\left\{\left(m_{t} n \cdot p_{2}\right)\left[\left(p_{1} \cdot q_{2}\right)\left(\left(k_{2}-p_{1}\right) \cdot q_{1}\right)-\left(\left(k_{2}-p_{1}\right) \cdot q_{2}\right)\left(p_{1} \cdot q_{1}\right)\right]-\left(m_{t} n \cdot q_{2}\right)\left[\left(p_{1} \cdot p_{2}\right)\left(\left(k_{2}-p_{1}\right) \cdot q_{1}\right)\right.\right. \\
& \left.-\left(\left(k_{2}-p_{1}\right) \cdot p_{2}\right)\left(p_{1} \cdot q_{1}\right)\right]+\left(m_{t} n \cdot q_{1}\right)\left[\left(p_{1} \cdot p_{2}\right)\left(\left(k_{2}-p_{1}\right) \cdot q_{2}\right)-\left(\left(k_{2}-p_{1}\right) \cdot p_{2}\right)\left(p_{1} \cdot q_{2}\right)\right]+m_{t}^{2}\left[\left(q_{2} \cdot p_{2}\right)\left(q_{1} \cdot\left(k_{2}-p_{1}\right)\right)\right. \\
& \left.+\left(q_{1} \cdot p_{2}\right)\left(q_{2} \cdot\left(k_{2}-p_{1}\right)\right)-\left(q_{1} \cdot q_{2}\right)\left(p_{2} \cdot\left(k_{2}-p_{1}\right)\right)\right] \pm m_{t}\left(n \cdot\left(k_{2}-p_{1}\right)\right)\left[\left(q_{2} \cdot p_{2}\right)\left(q_{1} \cdot p_{1}\right)+\left(q_{1} \cdot p_{2}\right)\left(q_{2} \cdot p_{1}\right)\right. \\
& \left.\left.-\left(q_{1} \cdot q_{2}\right)\left(p_{2} \cdot p_{1}\right)\right] \mp m_{t}\left(p_{1} \cdot\left(k_{2}-p_{1}\right)\right)\left[\left(q_{2} \cdot p_{2}\right)\left(q_{1} \cdot n\right)+\left(q_{1} \cdot p_{2}\right)\left(q_{2} \cdot n\right)-\left(q_{1} \cdot q_{2}\right)\left(p_{2} \cdot n\right)\right]\right\},
\end{aligned}
$$

and

$$
\begin{aligned}
A_{m_{b}}^{( \pm)}= & \frac{m_{b}|g|^{4}\left|K_{u d}\right|^{2}}{\left(k_{2}^{2}-M_{W}^{2}\right)^{2}} \frac{g_{ \pm}^{*} g_{\mp}}{2}\left\{2\left(p_{1} \cdot p_{2}\right)\left[\left(n \cdot q_{2}\right)\left(\left(k_{1}-p_{1}\right) \cdot q_{1}\right)-\left(n \cdot q_{1}\right)\left(\left(k_{1}-p_{1}\right) \cdot q_{2}\right)\right]-2\left(n \cdot p_{2}\right)\left[\left(p_{1} \cdot q_{2}\right)\left(\left(k_{1}-p_{1}\right) \cdot q_{1}\right)\right.\right. \\
& \left.-\left(p_{1} \cdot q_{1}\right)\left(\left(k_{1}-p_{1}\right) \cdot q_{2}\right)\right] \pm i \varepsilon^{\mu \nu \alpha \beta} q_{2 \alpha} q_{1 \beta}\left(n_{\mu} p_{1 \nu}\left(k_{1}-p_{1}\right) \cdot p_{2}+p_{2 \mu} n_{\nu}\left(k_{1}-p_{1}\right) \cdot p_{1}+p_{1 \mu} p_{2 \nu}\left(k_{1}-p_{1}\right) \cdot n\right) \\
& \mp i \varepsilon^{\mu \nu \alpha \beta} q_{2 \alpha} q_{1 \beta}\left(k_{1}-p_{1}\right) \mu\left[n_{\nu}\left(p_{1} \cdot\left(k_{2}-p_{1}\right)\right)+\left(k_{2}-p_{1}\right)_{\nu}\left(p_{1} \cdot n\right)\right]+\left(n \cdot\left(k_{1}-p_{1}\right)\right)\left[\left(p_{1} \cdot q_{2}\right)\left(\left(k_{2}-p_{1}\right) \cdot q_{1}\right)\right. \\
& \left.-\left(\left(k_{2}-p_{1}\right) \cdot q_{2}\right)\left(p_{1} \cdot q_{1}\right)\right]-\left(n \cdot q_{2}\right)\left[\left(p_{1} \cdot\left(k_{1}-p_{1}\right)\right)\left(\left(k_{2}-p_{1}\right) \cdot q_{1}\right)-\left(\left(k_{2}-p_{1}\right) \cdot\left(k_{1}-p_{1}\right)\right)\left(p_{1} \cdot q_{1}\right)\right]+\left(n \cdot q_{1}\right) \\
& \times\left[\left(p_{1} \cdot\left(k_{1}-p_{1}\right)\right)\left(\left(k_{2}-p_{1}\right) \cdot q_{2}\right)-\left(\left(k_{2}-p_{1}\right) \cdot\left(k_{1}-p_{1}\right)\right)\left(p_{1} \cdot q_{2}\right)\right]+2 m_{t}\left[\left(q_{2} \cdot\left(k_{1}-p_{1}\right)\right)\left(q_{1} \cdot p_{2}\right)+\left(q_{1} \cdot\left(k_{1}-p_{1}\right)\right)\right. \\
& \left.\left.\times\left(q_{2} \cdot p_{2}\right)-\left(q_{1} \cdot k_{1}\right)\left(q_{2} \cdot k_{1}\right)\right]+m_{t}\left(q_{1} \cdot q_{2}\right)\left[\left(p_{2} \cdot p_{1}\right)+\left(\left(k_{1}-p_{1}\right) \cdot\left(k_{1}-p_{2}\right)\right)\right]\right\}+m_{b}^{2} \frac{\left|g_{ \pm}\right|^{2}}{2} \frac{|g|^{4}\left|K_{u d}\right|^{2}\left|K_{t b}\right|^{2}}{\left(k_{2}^{2}-M_{W}^{2}\right)^{2}} \\
& \times\left\{-m_{t}\left[\left(n \cdot q_{2}\right)\left(p_{1} \cdot q_{1}\right)-\left(n \cdot q_{1}\right)\left(p_{1} \cdot q_{2}\right)\right]+m_{t}^{2}\left(q_{1} \cdot q_{2}\right)-2\left[\left(q_{2} \cdot\left(k_{1}-p_{1}\right)\right)\left(q_{1} \cdot \frac{p_{1} \mp m_{t} n}{2}\right)\right.\right. \\
& \left.\left.+\left(q_{1} \cdot\left(k_{1}-p_{1}\right)\right)\left(q_{2} \cdot \frac{p_{1} \mp m_{t} n}{2}\right)-\left(q_{1} \cdot q_{2}\right)\left(\left(k_{1}-p_{1}\right) \cdot \frac{p_{1} \mp m_{t} n}{2}\right)\right]\right\} .
\end{aligned}
$$

Finally, it can be shown that we can obtain the other matrix elements from the above expressions performing the following changes:

$$
\begin{gathered}
\left|M_{-}^{u}\right|^{2} \leftrightarrow\left|M_{+}^{\bar{u}}\right|^{2} \Leftrightarrow n \leftrightarrow-n, \\
\left|M_{-}^{u}\right|^{2} \leftrightarrow\left|M_{+}^{d}\right|^{2} \Leftrightarrow g_{L} \leftrightarrow g_{R}^{*}, \\
\left|M_{-}^{u}\right|^{2} \leftrightarrow\left|M_{-}^{\bar{d}}\right|^{2} \Leftrightarrow q_{1} \leftrightarrow q_{2} .
\end{gathered}
$$

It is useful to note also that all matrix elements are symmetric under the change

$$
\left(n, g_{L}, q_{1}\right) \leftrightarrow\left(-n, g_{R}^{*}, q_{2}\right) .
$$


[1] A.N. Okpara, hep-ph/0105151 and Proceedings for the 36th Rencontres de Moriond, QCD and High Energy Hadronic Interactions, Les Arcs, France, 2001.

[2] M.S. Chanowitz, Phys. Rev. D 59, 073005 (1999); Phys. Rev. Lett. 87, 231802 (2001).

[3] T. Appelquist and C. Bernard, Phys. Rev. D 22, 200 (1980); A. Longhitano, ibid. 22, 1166 (1980); A. Longhitano, Nucl. Phys. B188, 118 (1981); R. Renken and M. Peskin, ibid. B211, 93 (1983); A. Dobado, D. Espriu, and M.J. Herrero, Phys. Lett. B 255, 405 (1991).

[4] T. Appelquist, M. Bowick, E. Cohler, and A. Hauser, Phys. Rev. D 31, 1676 (1985).

[5] C.P. Burgess, S. Godfrey, H. Konig, D. London, and I. Maksymyk, Phys. Rev. D 49, 6115 (1994); E. Malkawi and C.P. Yuan, ibid. 50, 4462 (1994); 52, 472 (1995).

[6] See, e.g., S. Catani, M. Mangano, P. Nason, and L. Trentadue, Phys. Lett. B 378, 329 (1996).

[7] T.M.P. Tait, Ph. D. thesis, Michigan State University, 1999, hep-ph/9907462; T.M.P. Tait, Phys. Rev. D 61, 034001 (2000); T.M.P. Tait and C.-P. Yuan, ibid. 63, 014018 (2001); hep-ph/9710372.

[8] S. Dawson, Nucl. Phys. B249, 42 (1985); S. Willenbrock and D. Dicus, Phys. Rev. D 34, 155 (1986); C.P. Yuan, ibid. 41, 42 (1990); R.K. Ellis and S. Parke, ibid. 46, 3785 (1992); G. Bordes and B. van Eijk, Z. Phys. C 57, 81 (1993); Nucl. Phys. B435, 23 (1995); D.O. Carlson, Ph. D. thesis, Michigan State University, 1995, hep-ph/9508278; T. Stelzer, Z. Sullivan, and S. Willenbrock, Phys. Rev. D 56, 5919 (1997).

[9] T. Stelzer, Z. Sullivan, and S. Willenbrock, Phys. Rev. D 56, 5919 (1997); 58, 094021 (1998).
[10] F. Larios and C.P. Yuan, Phys. Rev. D 55, 7218 (1997).

[11] G. Mahlon and S. Parke, Phys. Rev. D 55, 7249 (1997); Phys. Lett. B 476, 323 (2000).

[12] E. Bagan, D. Espriu, and J. Manzano, Phys. Rev. D 60, 114035 (1999).

[13] D. Espriu and J. Manzano, Phys. Rev. D 63, 073008 (2001).

[14] Particle Data Group, C. Caso et al., Eur. Phys. J. C 3, 1 (1998).

[15] D. Amidei and C. Brock, "Report of the the TeV2000 study group on future electroweak physics at the Tevatron," FERMILAB-PUB-96-082, 1996.

[16] CLEO Collaboration, T. Swarnicki, in Proceedings of the 29th International Conference on High-Energy Physics, Vancouver, 1998 (World Scientific, Singapore, 1999), Vol. 2, p. 1057.

[17] F. Larios, M.A. Perez, and C.P. Yuan, Phys. Lett. B 457, 334 (1999); F. Larios, E. Malkawi, and C.P. Yuan, Acta Phys. Pol. B 27, 3741 (1996).

[18] G. D'Ambrosio and D. Espriu (in preparation).

[19] CTEQ4 Collaboration, H.-L. Lai et al., Phys. Rev. D 55, 1280 (1997).

[20] S. Parke, Proceedings of the International Symposium on Large QCD Corrections and New Physics, Hiroshima, 1997, Fermilab-Conf-97-431-T, hep-ph/9712512.

[21] G.P. Lepage, J. Comput. Phys. 27, 192 (1978).

[22] M. Jezabek and J.H. Kühn, Phys. Lett. B 329, 317 (1994).

[23] M. Fischer, S. Groote, J. G. Körner, and M. C. Mauser, Phys. Rev. D 65, 054036 (2002).

[24] D. Espriu and J. Manzano, in Proceedings of the 29 International Meeting on Fundamental Physics, Sitges, 2001, edited by A. Dobado and V. Fonseca, hep-ph/0109059.

[25] S. Dawson, Nucl. Phys. B249, 42 (1985). 